\title{
Diastereoselective Osmium-catalyzed Vicinal Oxyamination of Acyclic Allylic Alcohol Derivatives
}

\author{
Masruri, ${ }^{1,2}$ Nicholas Kanizaj, ${ }^{1}$ and Malcolm D. McLeod ${ }^{1 *}$ \\ ${ }^{1}$ Research School of Chemistry, Australian National University, Canberra, ACT 0200, Australia \\ ${ }^{2}$ Department of Chemistry, University of Brawijaya, Malang, Indonesia
}

Short title: Diastereoselective Oxyamination

Key words: aminohydroxylation, amino alcohol, nitrogen source, alkene, catalyst, regioselective

Assoc. Prof. Malcolm Donald McLeod

Research School of Chemistry, Australian National University, Canberra, ACT 0200, Australia

Phone: +61 261253405

Fax: +61 261258114

Email: malcolm.mcleod@anu.edu.au

\begin{abstract}
The osmium-catalyzed oxyamination of chiral acyclic allylic alcohol derivatives bearing mono- and 1,1-di-substituted double bonds with benzyl $N$-(4-tosyloxy)carbamate proceeds with high regioselectivity and moderate levels of diastereoselectivity favoring the anti product. The observed stereoselectivity shows a clear and systematic trend with anti:syn ratios increasing in line with the size of substituent at both the allylic stereocenter and double bond $\alpha$-carbon. The stereoinduction is in accord with the sense of diastereoselectivity predicted by Kishi's empirical rule and a previously reported transition state model for the osmium-catalyzed dihydroxylation of allylic alcohol derivatives. In contrast, allylic alcohol derivatives bearing trisubstituted double bonds show low or no reactivity in the oxyamination reaction affording the syn product in low yield in the cases examined.
\end{abstract}


Diastereoselective Oxyamination 


\section{Introduction}

The osmium-catalyzed oxyamination of alkenes is among the most powerful and general of methods for the synthesis of the vicinal amino alcohol functional array ${ }^{1,2}$ and is typified by the catalytic Asymmetric Aminohydroxylation (AA) reaction developed by Sharpless and coworkers. ${ }^{3,4}$ The recent development of pre-formed nitrogen sources has improved the efficiency, chemoselectivity and control of the osmium-catalyzed oxyamination reaction. Advances include the development of the intramolecular Tethered Aminohydroxylation (TA) reaction ${ }^{5}$ of carbamates ${ }^{6,7}$ and amides ${ }^{8}$ that provides secure control over regioselectivity. The development of non-tethered pre-formed nitrogen sources has further expanded the scope of this process, ${ }^{9-12}$ and includes the introduction of $\mathrm{N}$-(4-chlorobenzoyloxy)carbamates which allow for the enantioselective intermolecular oxyamination of mono- and di-substituted alkenes in the presence of cinchona alkaloid-derived chiral ligands. ${ }^{9,10}$ We recently reported the development of $N$-(4tosyloxy)carbamates as pre-formed nitrogen sources in the osmium-catalyzed vicinal oxyamination reaction of a range of mono-, di- and tri-substituted alkenes. ${ }^{12}$ The reactions occur with low catalyst loadings and afford good regioselectivity for unsymmetrically substituted alkenes. However, preliminary investigations showed that these nitrogen sources could not be directly substituted for the $N$-chlorocarbamates in the Sharpless AA reaction.

The diastereoselectivity of the osmium-catalyzed oxyamination reaction of chiral alkenes is also of importance for the stereoselective synthesis of amino-alcohol containing products, either in its own right, or as a complicating factor in reactions promoted by chiral ligands. Several reports of diastereoselectivity the intermolecular oxyamination reaction serve to highlight the potential of this element of stereocontrol for the synthesis of amino alcohol containing compounds, ${ }^{4,13,14}$ including natural products. ${ }^{15-17}$ Despite these disclosures there have been few systematic investigations of the influence of diastereoselectivity in the oxyamination reaction. Recently, a study reported the influence of exocyclic stereochemistry on the on the intramolecular osmium-catalyzed TA reaction. ${ }^{14}$ Herein we disclose a study on the diastereoselectivity of the osmium-catalyzed intermolecular vicinal oxyamination of acyclic allylic alcohol derivatives with benzyl $N$-(4tosyloxy)carbamate (1, Scheme 1). These reactions proceed with high regioselectivity and moderate levels of diastereoselectivity favoring the anti product for substrates bearing mono- and 1,1-disubstituted double bonds. The observed stereoselectivity shows a clear and systematic trend that varies with substrate substitution patterns. The stereoinduction is in accord with the sense of diastereoselectivity predicted by Kishi's empirical rule ${ }^{18,19}$ and conforms to a previously reported transition state model for the osmium-catalyzed dihydroxylation of allylic alcohol derivatives. ${ }^{20}$ In 
contrast, allylic alcohol derivatives bearing trisubstituted double bonds show low or no reactivity in the oxyamination reaction, affording the syn product in the two successful examples studied.

<Scheme 1 here $>$

Scheme 1. The oxyamination of acyclic allylic alcohol derivatives.

\section{Materials and Methods}

The general materials and methods are as previously reported. ${ }^{12}$

\section{General procedure 1 for the osmium-catalyzed oxyamination reaction}

To a stirred solution of allylic alcohol or derivative (1.0 equiv) and potassium osmate dihydrate (0.04 equiv) in acetonitrile/water (1:1, $5 \mathrm{~mL} \mathrm{mmol}^{-1}$ allylic alcohol or derivative) was added a solution of benzyl $N$-(4-tosyloxy)carbamate (1) ${ }^{12}$ (1.2 equiv) in acetonitrile (5 mL mmol ${ }^{-1}$ allylic alcohol or derivative) at $0{ }^{\circ} \mathrm{C}$. The reaction mixture was stirred at room temperature until the reaction was complete. The reaction was quenched with aqueous sodium hydrogen sulfite (5.00 ml, $0.05 \mathrm{M}$ ) and then stirred for $30 \mathrm{~min}$. The mixture was filtered through celite and extracted with ethyl acetate $(3 \times 10 \mathrm{ml})$. The combined organic extract was washed with brine $(10 \mathrm{ml})$, dried over sodium sulfate and concentrated under reduced pressure to afford the crude product. Purification by flash chromatography provided the target compounds.

\section{General procedure 2 for acetonide formation}

To a stirred solution of diol (1.0 equiv) in dichloromethane (3 ml) and 2,2-dimethoxypropane (4.0 equiv) at room temperature was added camphorsulfonic acid (0.04 equiv) and p-toluenesulfonic acid (0.04 equiv). The reaction mixture was stirred until the reaction was complete. The reaction was quenched with aqueous sodium hydrogen carbonate $(3.0 \mathrm{ml}, 1.0 \mathrm{M})$, and extracted with dichloromethane $(3 \times 5 \mathrm{ml})$. The combined organic extract was washed with brine $(5 \mathrm{ml})$, dried over sodium sulfate and concentrated under reduced pressure. Purification by flash chromatography afforded the target compounds.

rac-(2R,3S)-4-(Benzyloxycarbonylamino)-3-hydroxybutan-2-yl acetate (4a) and rac-(2R,3R)-4(benzyloxycarbonylamino)-3-hydroxybutan-2-yl acetate (6a)

The reaction was conducted according to general procedure 1 with $r a c$-but-3-en-2-yl acetate (2a) ${ }^{21}$ (60.0 mg, $0.53 \mathrm{mmol}$ ), potassium osmate dihydrate (7.00 mg, $0.02 \mathrm{mmol})$ and benzyl $\mathrm{N}$-(4- 
tosyloxy)carbamate (1) (221 mg, 0.69 mmol). Purification by flash chromatography (30\% ethyl acetate/n-hexane) afforded rac-(2R,3S)-4-(benzyloxycarbonylamino)-3-hydroxybutan-2-yl acetate (4a) as a colorless oil (42.4 mg, 29\%), $R_{f} 0.44$ (70\% ethyl acetate/ $n$-hexane). IR (thin film, $\mathrm{cm}^{-1}$ ) 3369 (O-H, N-H), 3066, 3034, 2981, 2925, 2825 (C-H), 1717 (C=O), 1533. ' H-NMR (400 MHz, $\left.\mathrm{CDCl}_{3}\right)$ 7.29-7.38 (5H, m, ArH), 5.29 (1H, s, NH), 5.07-5.13 (2H, m, CH $\mathrm{CH}_{2}$ ), 4.90 (1H, dq, J 6.1, $6.1 \mathrm{~Hz}, \mathrm{H} 2), 3.70$ (1H, m, H3), 3.50 (1H, ddd, J 14.0, 6.8, 2.8 Hz, H4A), 3.11 (1H, m, H4B), 3.01 (1H, s, OH), 2.05 (3H, s, CH $), 1.27$ (3H, d, J 6.8 Hz, H1). ${ }^{13} \mathrm{C}-\mathrm{NMR}\left(100 \mathrm{MHz}, \mathrm{CDCl}_{3}\right)$ 170.83, 156.93, 136.26, 128.54, 128.19, 128.11, 73.08, 71.56, 66.96, 43.48, 21.19, 16.23. LRMS (ESI+) 304 ([M+Na] $\left.]^{+}, 100 \%\right), 238$ (5), 178 (5), 120 (6), 91 (64). HRMS (ESI+) calcd for $\mathrm{C}_{14} \mathrm{H}_{20} \mathrm{NO}_{5}$ $\left([\mathrm{M}+\mathrm{H}]^{+}\right)$282.1341, found 282.1339; calcd for $\mathrm{C}_{14} \mathrm{H}_{19} \mathrm{NO}_{5} \mathrm{Na}\left([\mathrm{M}+\mathrm{Na}]^{+}\right) 304.1161$, found 304.1159.

A second fraction afforded rac-(2R,3R)-4-(benzyloxycarbonylamino)-3-hydroxybutan-2-yl acetate (6a) as a colorless oil (36.6 mg, 25\%), $R_{f} 0.40$ (70\% ethyl acetate/n-hexane). IR (thin film, $\mathrm{cm}^{-1}$ ) 3400 (O-H, N-H), 3066, 3034, 2982, 2940 (C-H), 1721 (C=O), 1533. ${ }^{1} \mathrm{H}-\mathrm{NMR}\left(400 \mathrm{MHz}, \mathrm{CDCl}_{3}\right)$ 7.30-7.38 (5H, m, ArH), 5.24 (1H, s, NH), 5.10 (2H, s, CH2Ar), 4.91 (1H, dq, J 6.1, 6.1 Hz, H2), 3.74 (1H, m, H3), 3.66 (1H, m, H4A), 3.28 (1H, m, H4B), 2.60 (1H, s, OH), 2.08 (3H, s, CH $\mathrm{CH}_{3}, 1.28$ (3H, d, J 6.8 Hz, H1). ${ }^{13} \mathrm{C}-\mathrm{NMR}\left(100 \mathrm{MHz}, \mathrm{CDCl}_{3}\right)$ 170.84, 157.48, 136.22, 128.54, 128.20, 128.12, 73.28, 71.62, 67.05, 42.95, 21.21, 15.63. LRMS (ESI+) 304 ([M+Na]+, 100\%), 218 (4), 120 (6), 91 (56). HRMS (ESI+) calcd for $\mathrm{C}_{14} \mathrm{H}_{19} \mathrm{NO}_{5} \mathrm{Na}\left([\mathrm{M}+\mathrm{Na}]^{+}\right)$304.1161, found 304.1161.

\section{rac-Benzyl (2S,3R)-2,3-dihydroxybutylcarbamate (5a) and rac-benzyl (2R,3R)-2,3- dihydroxybutylcarbamate (7a)}

Procedure 1: The reaction was conducted according to general procedure 1 with rac-3-buten-2-ol (3a) (45.9 mg, $0.64 \mathrm{mmol})$, potassium osmate dihydrate (9.40 mg, $0.03 \mathrm{mmol})$ and benzyl $\mathrm{N}$-(4tosyloxy)carbamate (1) (246 mg, $0.76 \mathrm{mmol}$ ). Integration of the $\mathrm{NH}$ signal in the $400 \mathrm{MHz}{ }^{1} \mathrm{H}$ NMR spectrum of the crude reaction mixture provided a 1.8:1 ratio of rac-benzyl (2S,3R)-2,3dihydroxybutylcarbamate (5a) [ $\delta 5.46(1 \mathrm{H}, \mathrm{s}, \mathrm{NH})]$ and rac-benzyl $(2 R, 3 R)-2,3-$ dihydroxybutylcarbamate (7a) $[\delta 5.37(1 \mathrm{H}, \mathrm{s}, \mathrm{NH})]$. Purification by flash chromatography (gradient from $50 \%$ to $100 \%$ ethyl acetate/ $n$-hexane) afforded an inseparable mixture of two diastereomers as a colorless oil (121 mg, 79\%), $R_{f} 0.11$ (60\% ethyl acetate/n-hexane). HRMS (ESI+) calcd for $\mathrm{C}_{12} \mathrm{H}_{17} \mathrm{NO}_{4} \mathrm{Na}\left([\mathrm{M}+\mathrm{Na}]^{+}\right)$262.1055, found 262.1049.

Procedure 2: To a solution of rac-(2R,3S)-4-(benzyloxycarbonylamino)-3-hydroxybutan-2-yl acetate (4a) (20.0 mg, $0.07 \mathrm{mmol}$ ) was added a solution of potassium carbonate (23.1 mg, 0.17 
$\mathrm{mmol})$ in methanol $(3 \mathrm{ml})$ and water $(1 \mathrm{ml})$ at $20^{\circ} \mathrm{C}$. This mixture was stirred at room temperature until the reaction was complete $(2 \mathrm{~h})$. The reaction was quenched with aqueous hydrochloric acid $(1.0 \mathrm{ml}, 1.0 \mathrm{M})$ and extracted with dichloromethane $(3 \times 10 \mathrm{ml})$. The combined organic extract was washed with brine $(5 \mathrm{ml})$ dried over sodium sulfate and concentrated under reduced pressure to afford rac-benzyl (2S,3R)-2,3-dihydroxybutylcarbamate (5a) as a colorless oil (17.0 mg, 100\%), $R_{f}$ 0.18 (70\% ethyl acetate/n-hexane). IR (thin film, $\mathrm{cm}^{-1}$ ) $3341(\mathrm{O}-\mathrm{H}), 3065,3034,2970,2926(\mathrm{C}-\mathrm{H})$, 1695 (C=O), 1534. ${ }^{1} \mathrm{H}-\mathrm{NMR}\left(400 \mathrm{MHz}, \mathrm{CDCl}_{3}\right)$ 7.30-7.38 (5H, m, ArH), 5.30 (1H, s, NH), 5.11 (2H, s, $\mathrm{CH}_{2} \mathrm{Ar}$ ), 3.75 (1H, m, H3), 3.55 (1H, m, H2), 3.35-3.43 (2H, m, H1), 2.79 (1H, s, OH), 1.70 (1H, s, OH), 1.22 (3H, d, J 6.4 Hz, H4). ${ }^{13} \mathrm{C}-\mathrm{NMR}\left(100 \mathrm{MHz}, \mathrm{CDCl}_{3}\right)$ 157.90, 136.16, 128.55, 128.25, 128.13, 75.05, 68.40, 67.15, 42.83, 18.41. LRMS (ESI+) 262 ([M+Na] $\left.]^{+}, 62 \%\right), 259$ (6), 196 (10), 181 (5), 120 (4), 91 (100), 60 (6). HRMS (ESI+) calcd for $\mathrm{C}_{12} \mathrm{H}_{17} \mathrm{NO}_{4} \mathrm{Na}\left([\mathrm{M}+\mathrm{Na}]^{+}\right)$ 262.1055, found 262.1053.

\section{rac-Benzyl [(4S,5R)-2,2,5-trimethyl-1,3-dioxolan-4-yl]methylcarbamate (17)}

The reaction was conducted according to general procedure 2 with rac-benzyl (2S,3R)-2,3dihydroxybutylcarbamate (5a) (16.4 mg, $0.07 \mathrm{mmol}$ ), 2,2-dimethoxypropane (35.7 mg, $0.34 \mathrm{mmol}$ ), camphorsulfonic acid (0.600 mg, $0.003 \mathrm{mmol}$ ) and p-toluenesulfonic acid (0.500 mg, $0.003 \mathrm{mmol}$ ). Purification by flash chromatography (20\% ethyl acetate/ $n$-hexane) afforded rac-benzyl [(4S,5R)2,2,5-trimethyl-1,3-dioxolan-4-yl]methylcarbamate (17) as a colorless oil (10.1 mg, 53\%). IR (thin film, $\mathrm{cm}^{-1}$ ) 3340 (N-H), 3065, 3034, 2985, 2936 (C-H), 1722 (C=O), 1532. ${ }^{1} \mathrm{H}-\mathrm{NMR}$ (400 MHz, $\left.\mathrm{CDCl}_{3}\right)_{7} 7.30-7.37$ (5H, m, ArH), 5.11 (2H, s, CH $2 \mathrm{Ar}$ ), 5.07 (1H, s, NH), 4.33 (1H, dq, J 6.3, $6.3 \mathrm{~Hz}$, H5), 4.11 (1H, m, H4), 3.48 (1H, m, $\left.\underline{C}_{A} H_{B} N H\right), 3.05$ (1H, m, $\left.\mathrm{CH}_{A} \underline{H}_{B} \mathrm{NH}\right), 1.44$ (3H, s, $\left.\mathrm{CH}_{3}\right), 1.33$ (3H, s, $\mathrm{CH}_{3}$ ), 1.23 (3H, d, J $\left.6.4 \mathrm{~Hz}, \mathrm{CH}_{3}\right) .{ }^{13} \mathrm{C}-\mathrm{NMR}$ (100 MHz, $\mathrm{CDCl}_{3}$ ) 156.39, 136.45, 128.49, 128.11, 128.10, 108.09, 76.63, 72.61, 66.80, 41.59, 28.26, 25.51, 14.45. LRMS (ESI+) 302 ([M+Na] $\left.]^{+}, 88 \%\right), 222$ (5), 181 (6), 149 (4), 91 (100). HRMS (ESI+) calcd for $\mathrm{C}_{15} \mathrm{H}_{21} \mathrm{NO}_{4} \mathrm{Na}$ $([\mathrm{M}+\mathrm{Na}]+)$ 302.1368, found 302.1368.

rac-(2S,3R)-1-(Benzyloxycarbonylamino)-2-hydroxypentan-3-yl acetate (4b) and rac-(2R,3R)1-(benzyloxycarbonylamino)-2-hydroxypentan-3-yl acetate (6b)

The reaction was conducted according to general procedure 1 with rac-pent-1-en-3-yl acetate (2b) ${ }^{22}$ (53.9 mg, $0.42 \mathrm{mmol})$, potassium osmate dihydrate $(6.00 \mathrm{mg}, 0.02 \mathrm{mmol})$ and benzyl $\mathrm{N}$-(4tosyloxy)carbamate (1) (165 mg, $0.51 \mathrm{mmol}$ ). Purification by flash chromatography (40\% ethyl acetate/n-hexane) afforded rac-(2S,3R)-1-(benzyloxycarbonylamino)-2-hydroxypentan-3-yl acetate (4b) as a colorless oil (53.1 mg, 43\%), $R_{f} 0.46$ (65\% ethyl acetate/n-hexane). IR (thin film, $\mathrm{cm}^{-1}$ ) 
3400 (O-H, N-H), 3066, 3034, 2935, 2882, 2853 (C-H), 1713, 1701 (C=O), 1537. ${ }^{1} \mathrm{H}-\mathrm{NMR}(400$ $\mathrm{MHz}_{\mathrm{CDCl}}$ ) 7.30-7.36 (5H, m, ArH), 5.29 (1H, s, NH), 5.07-5.14 (2H, m, $\left.\mathrm{CH}_{2} \mathrm{Ar}\right), 4.82$ (1H, m, H3), 3.73 (1H, m, H2), 3.54 (1H, m, H1A), 3.06 (1H, ddd, J 14.0, 6.8, 4.8 Hz, H1B), 2.99 (1H, s, $\mathrm{OH}), 2.08$ (3H, s, $\left.\mathrm{CH}_{3}\right), 1.62-1.76$ (2H, m, H4), 0.93 (3H, t, J 7.2 Hz, H5). ${ }^{13} \mathrm{C}-\mathrm{NMR}(400 \mathrm{MHz}$, $\left.\mathrm{CDCl}_{3}\right)_{171.55}$ 157.63, 136.23, 128.53, 128.19, 128.11, 76.32, 72.26, 67.05, 42.90, 23.31, 21.01, 9.69. LRMS (ESI+) 318 ([M+Na]+, 100\%), 270 (5), 184 (6), 120 (8), 91 (68), 84 (8), 69 (4). HRMS (ESI+) calcd for $\mathrm{C}_{15} \mathrm{H}_{21} \mathrm{NO}_{5} \mathrm{Na}\left([\mathrm{M}+\mathrm{Na}]^{+}\right)$318.1317, found 318.1318.

A second fraction afforded rac-(2R,3R)-1-(benzyloxycarbonylamino)-2-hydroxypentan-3-yl acetate (6b) as a colorless oil (34.8 mg, 28\%), $R_{f} 0.36$ (65\% ethyl acetate/ $n$-hexane). IR (thin film, $\mathrm{cm}^{-1}$ ) 3369 (O-H, N-H), 3034, 2924, 2853 (C-H), 1713 (C=O), 1537. ํ--NMR (400 MHz, CDCl $)$ 7.297.38 (5H, m, ArH), 5.33 (1H, s, NH), 5.09-5.10 (2H, m, CH $2 \mathrm{Ar}), 4.81$ (1H, m, H3), 3.74 (1H, m, H2), 3.60 (1H, m, H1A), 3.26 (1H, m, H1B), 2.68 (1H, m, OH), 2.05-2.11 (3H, m, CH3), 1.62-1.72 (2H, m, H4), 0.90 (3H, t, $J 6.8$ Hz, H5). ${ }^{13} \mathrm{C}-\mathrm{NMR}\left(100 \mathrm{MHz}, \mathrm{CDCl}_{3}\right)$ 171.35, 157.22, 136.29, 128.53, 128.27, 128.17, 75.86, 71.52, 67.14, 43.71, 25.91, 20.99, 10.21. LRMS (ESI+) 318 ([M+Na] $\left.]^{+}, 90 \%\right), 91(100)$. HRMS (ESI+) calcd for $\mathrm{C}_{15} \mathrm{H}_{21} \mathrm{NO}_{5} \mathrm{Na}\left([\mathrm{M}+\mathrm{Na}]^{+}\right)$318.1317, found 318.1318.

\section{rac-Benzyl (2S,3R)-2,3-dihydroxypentylcarbamate (5b) and rac-benzyl (2R,3R)-2,3- dihydroxypentylcarbamate (7b)}

Procedure 1: The reaction was conducted according to general procedure 1 with rac-1-penten-3-ol (3b) $)^{22}$ (36.0 mg, $0.42 \mathrm{mmol}$ ), potassium osmate dihydrate (6.20 mg, $16.8 \mu \mathrm{mol}$ ) and benzyl $\mathrm{N}$-(4tosyloxy)carbamate (1) (148 mg, $0.46 \mathrm{mmol}$ ). Integration of the $\mathrm{NH}$ signals in the $400 \mathrm{MHz}{ }^{1} \mathrm{H}$ NMR spectrum of the crude reaction mixture provided a 2.1:1 ratio of rac-benzyl (2S,3R)-2,3dihydroxypentylcarbamate (5b) [ $\delta 5.32(1 \mathrm{H}, \mathrm{s}, \mathrm{NH})]$ and rac-benzyl $(2 R, 3 R)-2,3-$ dihydroxypentylcarbamate (7b) $[\delta 5.25(1 \mathrm{H}, \mathrm{s}, \mathrm{NH})]$. Purification by flash chromatography (60\% ethyl acetate/ $n$-hexane) afforded an inseparable a mixture of two diastereomers as a white solid (58.9 mg, 55\%), $R_{f} 0.19$ (60\% ethyl acetate/n-hexane). HRMS (ESI+) calcd for $\mathrm{C}_{13} \mathrm{H}_{19} \mathrm{NO}_{4} \mathrm{Na}$ $\left([\mathrm{M}+\mathrm{Na}]^{+}\right)$276.1212, found 276.1213.

Procedure 2: To a solution of rac-(2S,3R)-1-(benzyloxycarbonylamino)-2-hydroxypentan-3-yl acetate (4b) (20.0 mg, $0.07 \mathrm{mmol}$ ) was added a solution of potassium carbonate (23.1 mg, 0.17 $\mathrm{mmol})$ in methanol $(3.0 \mathrm{ml})$ and water $(1.0 \mathrm{ml})$ at $20^{\circ} \mathrm{C}$. This reaction mixture was stirred until the reaction was complete $(2 \mathrm{~h})$. The reaction was quenched with aqueous hydrochloric acid (1.0 ml, $1.0 \mathrm{M})$ and extracted with dichloromethane $(3 \times 10 \mathrm{ml})$. The combined organic extract was washed 
with brine ( $5 \mathrm{ml}$ ), dried over sodium sulfate and concentrated under reduced pressure to afford racbenzyl (2S,3R)-2,3-dihydroxypentylcarbamate (5b) as a colorless oil (15.1 mg, 88\%), $R_{f} 0.37$ (70\% ethyl acetate/n-hexane). IR (thin film, $\mathrm{cm}^{-1}$ ) 3330 (broad, O-H, N-H), 2961, 2926 (C-H), 1689 (C=O), 1547. ${ }^{1} \mathrm{H}-\mathrm{NMR}$ (400 MHz, CDCl 3 ) 7.30-7.37 (5H, m, ArH), 5.19 (1H, s, NH), 5.12 (2H, s, $\mathrm{CH}_{2} \mathrm{Ar}$ ), 3.59 (1H, m, H3), 3.39-3.49 (3H, m, H1 and H2), 2.58 (1H, s, OH), 2.51 (1H, s, OH), 1.62-1.43 (2H, m, H4), 1.01 (3H, t, J 7.2 Hz, H5). ${ }^{13} \mathrm{C}-\mathrm{NMR}$ (100 MHz, $\mathrm{CDCl}_{3}$ ) 157.94, 136.17, 128.54, 128.24, 128.13, 73.86, 73.78, 67.14, 42.83, 25.49, 10.14. LRMS (ESI+) $276\left([\mathrm{M}+\mathrm{Na}]^{+}\right.$, 100\%), 181 (7), 149 (12), 120 (4), 107 (5), 91 (68). HRMS (ESI+) calcd for $\mathrm{C}_{13} \mathrm{H}_{19} \mathrm{NO}_{4} \mathrm{Na}$ $\left([\mathrm{M}+\mathrm{Na}]^{+}\right)$276.1212, found 276.1212 .

rac-(2S,3R)-1-(Benzyloxycarbonylamino)-2-hydroxy-4-methylpentan-3-yl acetate (4c) and rac(2R,3R)-1-(benzyloxycarbonylamino)-2-hydroxy-4-methylpentan-3-yl acetate (6c)

The reaction was conducted according to general procedure 1 with rac-4-methylpent-1-en-3-yl acetate (2c) $)^{22}$ (60.0 mg, $0.42 \mathrm{mmol}$ ), potassium osmate dihydrate (6.2 mg, $0.02 \mathrm{mmol}$ ) and benzyl $N$-(4-tosyloxy)carbamate (1) (163 mg, $0.51 \mathrm{mmol}$ ). Purification by flash chromatography (30\% ethyl acetate/n-hexane) afforded rac-(2S,3R)-1-(benzyloxycarbonylamino)-2-hydroxy-4methylpentan-3-yl acetate (4c) as a colorless oil (62.2 mg, 48\%), $R_{f} 0.55$ (65\% ethyl acetate/nhexane). Further purification was conducted by HPLC (SunFire Prep Silica $5 \mu \mathrm{m}$, 3\% isopropanol 97\% $n$-hexane, flow rate $10 \mathrm{ml} / \mathrm{min}$ ) to give compound $4 \mathrm{c}\left(t_{\mathrm{R}} 8.9 \mathrm{~min}\right)$. IR (thin film, $\left.\mathrm{cm}^{-1}\right) 3407$ (O-H, N-H), 3090, 3066, 3034, 2965, 2938, 2878 (C-H), 1721 (C=O), 1530. ${ }^{1} \mathrm{H}-\mathrm{NMR}$ (400 MHz, $\left.\mathrm{CDCl}_{3}\right)$ 7.29-7.36 (5H, m, ArH), 5.35 (1H, s, NH), 5.06-5.15 (2H, m, CH $\left.2 \mathrm{Ar}\right), 4.75$ (1H, dd, J 6.8, 4.8 Hz, H3), 3.79 (1H, m, H2), 3.59 (1H, m, H1A), 2.99 (1H, ddd, J 14.4, 6.4, 4.8 Hz, H1B), 2.03$2.11\left(4 \mathrm{H}, \mathrm{m}, \mathrm{H} 4\right.$ and $\left.\mathrm{CH}_{3}\right), 1.66(1 \mathrm{H}, \mathrm{s}, \mathrm{OH}), 0.93-0.94\left(6 \mathrm{H}, \mathrm{m}, 2 \times \mathrm{CH}_{3}\right) .{ }^{13} \mathrm{C}-\mathrm{NMR}(100 \mathrm{MHz}$, $\left.\mathrm{CDCl}_{3}\right)$ 171.80, 157.89, 136.22, 128.52, 128.18, 128.10, 78.46, 70.53, 67.07, 42.99, 28.20, 20.88, 19.54, 16.65. LRMS (ESI+) 332 ([M+Na] , 98\%), 293 (10), 266 (5), 250 (6), 232 (7), 188 (8), 120 (11), 91 (100), 81 (8). HRMS (ESI+) calcd for $\mathrm{C}_{16} \mathrm{H}_{24} \mathrm{NO}_{5}\left([\mathrm{M}+\mathrm{H}]^{+}\right)$310.1654, found 310.1654; HRMS (ESI+) calcd for $\mathrm{C}_{16} \mathrm{H}_{23} \mathrm{NO}_{5} \mathrm{Na}\left([\mathrm{M}+\mathrm{Na}]^{+}\right)$332.1474, found 332.1474.

A second fraction afforded rac-(2R,3R)-1-(benzyloxycarbonylamino)-2-hydroxy-4-methylpentan-3yl acetate (6c) as a colorless oil (26.1 mg, 20\%), $R_{f} 0.29$ (65\% ethyl acetate/ $n$-hexane). Further purification was conducted by HPLC (SunFire Prep Silica $5 \mu \mathrm{m}$, 5\% isopropanol 95\% $n$-hexane, flow rate $10 \mathrm{ml} / \mathrm{min}$ ) to give compound $\mathbf{6 c}\left(t_{\mathrm{R}} 14.8 \mathrm{~min}\right)$. IR (thin film, $\left.\mathrm{cm}^{-1}\right) 3406(\mathrm{O}-\mathrm{H}, \mathrm{N}-\mathrm{H})$, 3065, 3033, 2966, 2877 (C-H), 1718 (C=O), 1528. ${ }^{1} \mathrm{H}-\mathrm{NMR}\left(400 \mathrm{MHz}, \mathrm{CDCl}_{3}\right.$ ) 7.30-7.36 (5H, m, ArH), 5.26 (1H, s, NH), 5.10 (2H, s, CH $\mathrm{CH}_{2}$, 4.64 (1H, dd, J 7.6, $\left.3.2 \mathrm{~Hz}, \mathrm{H} 3\right), 3.87$ (1H, m, H2), 
3.27 (1H, m, H1A), 3.14 (1H, m, H1B), 2.35 (1H, d, J 7.2 Hz, OH), 2.06-2.14 (4H, m, H4 and $\mathrm{CH}_{3}$ ), 0.95 (3H, d, $J 6.8 \mathrm{~Hz}, \mathrm{CH}_{3}$ ), 0.91 (3H, d, J 7.2 Hz, H5). ${ }^{13} \mathrm{C}-\mathrm{NMR}\left(100 \mathrm{MHz}, \mathrm{CDCl}_{3}\right)$ 171.49, 156.91, 136.29, 128.52, 128.16, 128.09, 78.99, 70.10, 66.92, 44.13, 28.61, 20.84, 19.08, 18.15. LRMS (ESI+) 332 ([M+Na] $\left.]^{+}, 100 \%\right), 246$ (5), 91 (21). HRMS (ESI+) calcd for $\mathrm{C}_{16} \mathrm{H}_{23} \mathrm{NO}_{5} \mathrm{Na}$ $\left([\mathrm{M}+\mathrm{Na}]^{+}\right)$332.1474, found 332.1473.

rac-Benzyl (2S,3R)-2,3-dihydroxy-4-methylpentylcarbamate (5c) and rac-benzyl (2R,3R)-2,3dihydroxy-4-methylpentylcarbamate (7c)

The reaction was conducted according to general procedure 1 with rac-4-methyl-1-penten-3-ol (3c) $)^{22}$ (41.7 mg, $0.42 \mathrm{mmol}$ ), potassium osmate dihydrate (6.1 mg, $\left.16.6 \mu \mathrm{mol}\right)$ and benzyl $\mathrm{N}$-(4tosyloxy)carbamate (1) (161 mg, $0.50 \mathrm{mmol}$ ). Integration of $\mathrm{NH}$ signal of $400 \mathrm{MHz}{ }^{1} \mathrm{H}-\mathrm{NMR}$ spectrum of the crude reaction mixture showed a 3.1:1 ratio of rac-benzyl (2S,3R)-2,3-dihydroxy-4methylpentylcarbamate (5c) and rac-benzyl (2R,3R)-2,3-dihydroxy-4-methylpentyl-carbamate (7c). Purification by flash chromatography (50\% ethyl acetate/ $n$-hexane) afforded an inseparable mixture of two diastereomers as a colorless oil (81.3 mg, 73\%), $R_{f} 0.22$ (5:2:3 ethyl acetate/dichloromethane/n-hexane). IR (thin film, $\mathrm{cm}^{-1}$ ) 3405 (O-H, N-H), 3067, 3034, 2960, 2930, 2875 (C-H), 1695 (C=O), 1532. ${ }^{1} \mathrm{H}-\mathrm{NMR}$ (400 MHz, CDCl $)$ 7.29-7.37 (5H, m, ArH), 5.40 (1H, $\mathrm{m}, \mathrm{NH}$ ) and 5.30* (1H, s, $\left.\mathrm{NH}^{*}\right), 5.07-5.14\left(2 \mathrm{H}, \mathrm{m}, \mathrm{CH}_{2} \mathrm{Ar}\right), 3.70$ (1H, m, H3), 3.36-3.42 (2H, m, H2 and H1A), 3.31 (1H, m, H1B), 2.88 (1H, s, OH), 2.76 (1H, s, OH), 1.82 (1H, m, H4), 0.96-0.95 $\left(6 \mathrm{H}, \mathrm{m}, 2 \times \mathrm{CH}_{3}\right)$ and $0.92^{*}\left(3 \mathrm{H}, \mathrm{d}, \mathrm{J} 6.8 \mathrm{~Hz}, \mathrm{CH}_{3}{ }^{*}\right) .{ }^{13} \mathrm{C}-\mathrm{NMR}\left(100 \mathrm{MHz}, \mathrm{CDCl}_{3}\right) 157.93\left(157.48^{*}\right)$, 136.22, 128.54, 128.22, 128.11, 77.16, 71.61 (70.64*), 67.09, 42.90 (44.41*), 29.31 (29.66*), 19.33, 17.10 (18.03*). LRMS (ESI+) $290\left([\mathrm{M}+\mathrm{Na}]^{+}, 100 \%\right), 91$ (38). HRMS (ESI+) calcd for $\mathrm{C}_{14} \mathrm{H}_{21} \mathrm{NO}_{4} \mathrm{Na}\left([\mathrm{M}+\mathrm{Na}]^{+}\right)$290.1368, found 290.1360. ( ${ }^{*}$ indicates peaks of the minor diastereomer).

\section{rac-Benzyl (2S,3R)-2-hydroxy-3-(4-methoxybenzyloxy)-2-methylbutylcarbamate (11a) and rac-benzyl (2R,3R)-2-hydroxy-3-(4-methoxybenzyloxy)-2-methylbutylcarbamate (14a)}

The reaction was conducted according to general procedure 1 with rac-1-methoxy-4-[(3-methylbut3-en-2-yloxy)methyl]benzene (8a) $)^{23}$ (35.0 mg, $0.17 \mathrm{mmol}$ ), potassium osmate dihydrate (2.50 mg, $0.01 \mathrm{mmol}$ ) and benzyl $\mathrm{N}$-(4-tosyloxy)carbamate (1) (65.4 mg, $0.20 \mathrm{mmol})$. Integration of the C2$\underline{\mathrm{C}}_{3}$ signals in the $400 \mathrm{MHz}{ }^{1} \mathrm{H}-\mathrm{NMR}$ spectrum of the crude reaction mixture showed a 2.6:1 ratio of rac-benzyl (2S,3R)-2-hydroxy-3-(4-methoxybenzyloxy)-2-methylbutylcarbamate (11a) [ $\delta 1.09$ $\left(3 \mathrm{H}, \mathrm{s}, \mathrm{CH}_{3}\right)$ ] and rac-benzyl (2R,3R)-2-hydroxy-3-(4-methoxybenzyloxy)-2-methylbutylcarbamate (14a) $\left[\delta 1.11\left(3 \mathrm{H}, \mathrm{s}, \mathrm{CH}_{3}\right)\right]$. Purification by flash chromatography (30\% ethyl acetate/n-hexane) afforded an inseparable mixture of two diastereomers as a yellow oil (43.6 mg, 69\%), $R_{f} 0.23$ (40\% 
ethyl acetate/n-hexane). Further purification by preparative HPLC ( $\mu$ Porasil silica $10 \mu \mathrm{m}, 3.9 \times 300$ $\mathrm{mm}$, solvent $1 \%$ isopropanol $/ n$-hexane, flow rate $0.7 \mathrm{ml} / \mathrm{min}$ ) afforded rac-benzyl (2R,3R)-2hydroxy-3-(4-methoxybenzyloxy)-2-methylbutylcarbamate $(\mathbf{1 4 a})\left(t_{\mathrm{R}} 31.8 \mathrm{~min}\right)$ as a colorless oil. IR (thin film, cm ${ }^{-1}$ ) 3419 (N-H, O-H), 3066, 3033, 2976, 2936, 2837 (C-H), 1717 (C=O), 1612, 1586 (C=C), 1514. ${ }^{1} \mathrm{H}-\mathrm{NMR}\left(400 \mathrm{MHz}, \mathrm{CDCl}_{3}\right)$ 7.29-7.36 (5H, m, ArH), 7.24 (2H, d, J 8.4 Hz, ArH), 6.87 (2H, d, J $8.4 \mathrm{~Hz}, \mathrm{ArH}), 5.10$ (3H, s, broad, $\mathrm{NH}$ and $\mathrm{CH}_{2}$ ), 4.60 (1H, d, J $10.8 \mathrm{~Hz}, \mathrm{C}_{\underline{\mathrm{A}}} \mathrm{H}_{\mathrm{B}} \mathrm{Ar}$ ), $4.31\left(1 \mathrm{H}, \mathrm{d}, J 11.2 \mathrm{~Hz}, \mathrm{CH}_{\mathrm{A}} \underline{\mathrm{H}}_{\mathrm{B}} \mathrm{Ar}\right), 3.79$ (3H, s, $\left.\mathrm{OCH}_{3}\right), 3.42$ (1H, q, $\left.J 6.4 \mathrm{~Hz}, \mathrm{H} 3\right), 3.31$ (1H, dd, $J$ 13.6, 5.6 Hz, H1A), 3.20 (1H, dd, J 13.6, 6.6 Hz, H1B), 2.65 (1H, s, OH), 1.19 (3H, d, J 6.4 Hz, H4), 1.09 (3H, s, $\left.\mathrm{CH}_{3}\right) .{ }^{13} \mathrm{C}-\mathrm{NMR}\left(100 \mathrm{MHz} \mathrm{CDCl}_{3}\right)$ 159.31, 156.82, 136.58, 130.14, 129.42, 128.48, 128.06, 128.01, 113.89, 77.95, 74.24, 70.79, 66.70, 55.24, 47.75, 20.22, 13.57. LRMS $(\mathrm{ESI}+) 396\left([\mathrm{M}+\mathrm{Na}]^{+}, 75 \%\right), 121(100) . \mathrm{HRMS}(\mathrm{ESI}+)$ calcd for $\mathrm{C}_{21} \mathrm{H}_{27} \mathrm{NO}_{5} \mathrm{Na}\left([\mathrm{M}+\mathrm{Na}]^{+}\right)$ 396.1787, found 396.1790.

A second fraction afforded rac-benzyl (2S,3R)-2-hydroxy-3-(4-methoxybenzyloxy)-2methylbutylcarbamate (11a) ( $t_{\mathrm{R}} 36.4 \mathrm{~min}$ ) as a colorless oil. IR (thin film, $\left.\mathrm{cm}^{-1}\right) 3418(\mathrm{~N}-\mathrm{H}, \mathrm{O}-\mathrm{H})$, 3065, 3033, 2975, 2936, 2874, 2837 (C-H), 1705 (C=O), 1612, 1586 (C=C), 1513. ${ }^{1} \mathrm{H}-\mathrm{NMR}$ (400 MHz, $\mathrm{CDCl}_{3}$ ) 7.29-7.38 (5H, m, ArH), 7.23 (2H, d, J 8.4 Hz, ArH), 6.86 (2H, d, J 8.8 Hz, ArH), 5.08 (3H, s, broad, $\mathrm{NH}$ and $\left.\mathrm{CH}_{2} \mathrm{Ar}\right), 4.60\left(1 \mathrm{H}, \mathrm{d}, J 10.8 \mathrm{~Hz}, \underline{\mathrm{C}}_{\mathrm{A}} \mathrm{H}_{\mathrm{B}} \mathrm{Ar}\right), 4.30$ (1H, d, $J 11.6 \mathrm{~Hz}$, $\left.\mathrm{CH}_{\mathrm{A}} \underline{\mathrm{H}}_{\mathrm{B}} \mathrm{Ar}\right), 3.77$ (3H, s, $\left.\mathrm{OCH}_{3}\right), 3.46$ (1H, q, J $\left.6.0 \mathrm{~Hz}, \mathrm{H} 3\right), 3.36$ (1H, dd, J 14.0, $\left.8.0 \mathrm{~Hz}, \mathrm{H} 1 \mathrm{~A}\right)$, 3.20 (1H, dd, J 13.6, 3.6 Hz, H1B), 2.89 (1H, s, OH), 1.21 (3H, d, J 6.0 Hz, H4), 1.11 (3H, s, CH $)$. ${ }^{13} \mathrm{C}-\mathrm{NMR}\left(100 \mathrm{MHz}, \mathrm{CDCl}_{3}\right)$ 159.32, 157.34, 136.55, 130.13, 129.39, 128.47, 128.03, 127.98, 113.92, 79.37, 73.98, 70.76, 66.73, 55.23, 46.88, 21.05, 13.50. LRMS (ESI+) $396\left([\mathrm{M}+\mathrm{Na}]^{+}, 75 \%\right)$, 121 (100). HRMS (ESI+) calcd for $\mathrm{C}_{21} \mathrm{H}_{27} \mathrm{NO}_{5} \mathrm{Na}\left([\mathrm{M}+\mathrm{Na}]^{+}\right)$396.1787, found 396.1788).

\section{rac-Benzyl (2S,3R)-2,3-dihydroxy-2-methylbutylcarbamate (13a)}

To a stirred solution of rac-benzyl (2S,3R)-2-hydroxy-3-(4-methoxybenzyloxy)-2methylbutylcarbamate (11a) (19 mg, $51 \mu \mathrm{mol}$ ) in a mixture of dichloromethane (459 $\mu \mathrm{l})$ and water $(51 \mu \mathrm{l})$ at $0{ }^{\circ} \mathrm{C}$ was added 2,3-dichloro-5,6-dicyano-1,4-benzoquinone (14 mg, $\left.61 \mu \mathrm{mol}\right)$. Following overnight stirring, the reaction mixture was diluted with dichloromethane $(1 \mathrm{ml})$. The reaction was quenched with saturated aqueous sodium hydrogen carbonate $(1.5 \mathrm{ml})$ and the crude product was extracted into ethyl acetate $(5 \times 1 \mathrm{ml})$. The combined organic extract was washed with saturated aqueous sodium hydrogen carbonate and brine, dried over magnesium sulfate and concentrated under reduced pressure to afford the crude product. Purification by flash chromatography (30 to 100\% ethyl acetate/40-60 petroleum spirit) afforded the title compound 13a as a colorless oil (4 
mg, 30\%). The identity was confirmed by ${ }^{1} \mathrm{H}$ and ${ }^{13} \mathrm{C}$ NMR comparison with authentic rac-benzyl (2S,3R)-2,3-dihydroxy-2-methylbutylcarbamate. ${ }^{12}$

\section{rac-1-Methoxy-4-[(2-methylpent-1-en-3-yloxy)-methyl]-benzene (8b)}

To a stirred solution of rac-2-methylpent-1-en-3-ol (10b) $)^{24}$ (800 mg, 7.99 mmol) and sodium hydride (383 $\mathrm{mg}, 16.0 \mathrm{mmol})$ in dichloromethane $(5.0 \mathrm{ml})$ at $0{ }^{\circ} \mathrm{C}$ under nitrogen was added $p$ methoxybenzyl chloride (1.38 g, $8.81 \mathrm{mmol})$. The reaction mixture was stirred at room temperature until the reaction was complete $(24 \mathrm{~h})$. The reaction was quenched with aqueous sodium hydrogen carbonate (5.0 ml, $1.0 \mathrm{M}$ ), and the layers were separated. The aqueous layer was further extracted with dichloromethane $(3 \times 5 \mathrm{ml})$. The combined organic extract was washed with brine $(10 \mathrm{ml})$, dried over sodium sulfate and concentrated under reduced pressure to afford the crude product. Purification by flash chromatography (2\% ethyl acetate/ $n$-hexane) afforded the title compound $\mathbf{8 b}$ as a colorless oil (1.18 mg, 67\%), $R_{f} 0.27$ (5\% ethyl acetate/ $n$-hexane). IR (thin film, $\mathrm{cm}^{-1}$ ) 3071, 3034, 2963, 2936, 2874, 2836 (C-H), 1649, 1613 (C=C), 1586, 1513. ${ }^{1} \mathrm{H}-\mathrm{NMR}\left(400 \mathrm{MHz}, \mathrm{CDCl}_{3}\right)$ 7.23-7.26 (2H, m, ArH), 6.84-6.87 (2H, m, ArH), 4.96-4.95 (1H, m, H1A), 4.89 (1H, m, H1B), $4.43\left(1 \mathrm{H}, \mathrm{d}, J 11.2 \mathrm{~Hz}, \mathrm{C}_{\underline{\mathrm{A}}} \mathrm{H}_{\mathrm{B}} \mathrm{Ar}\right), 4.18$ (1H, d, J $\left.11.6 \mathrm{~Hz}, \mathrm{CH}_{\mathrm{A}} \underline{\mathrm{H}_{\mathrm{B}} \mathrm{Ar}}\right), 3.79$ (3H, s, OCH $)$, 3.59 (1H, t, $J 6.8 \mathrm{~Hz}, \mathrm{H} 3), 1.42-1.68$ (5H, m, H4 and $\mathrm{CH}_{3}$ ), 0.89 (3H, t, $\left.J 7.6 \mathrm{~Hz}, \mathrm{H} 5\right) .{ }^{13} \mathrm{C}-\mathrm{NMR}(100$ $\mathrm{MHz}, \mathrm{CDCl}_{3}$ ) 158.99, 144.55, 130.99, 129.31, 113.72, 113.69, 84.55, 69.48, 55.24, 26.45, 16.46, 10.22. LRMS (EI+) 220 (M+•, 5\%), 137 (62), 121 (100), 109 (8), 91 (6), 84 (20), 77 (15), 69 (19), 65 (4), 55 (10). HRMS (EI+) calcd for $\mathrm{C}_{14} \mathrm{H}_{20} \mathrm{O}_{2}\left(\mathrm{M}^{+\bullet}\right)$ 220.1463, found 220.1466.

\section{rac-Benzyl (2S,3R)-2-hydroxy-3-(4-methoxybenzyloxy)-2-methylpentylcarbamate (11b) and rac-benzyl (2R,3R)-2-hydroxy-3-(4-methoxybenzyloxy)-2-methylpentylcarbamate (14b)}

The reaction was conducted according to general procedure 1 with rac-1-methoxy-4-[(2methylpent-1-en-3-yloxy)methyl]benzene (8b) (65.0 mg, $0.30 \mathrm{mmol}$ ), potassium osmate dihydrate (4.40 mg, $0.01 \mathrm{mmol}$ ) and benzyl $N$-(4-tosyloxy)carbamate (1) (114 mg, $0.35 \mathrm{mmol}$ ). Integration of the $\mathrm{C} 2-\mathrm{CH}_{3}$ signal of the $400 \mathrm{MHz}{ }^{1} \mathrm{H}-\mathrm{NMR}$ spectrum of the crude reaction mixture showed a 4.9:1 ratio of rac-benzyl (2S,3R)-2-hydroxy-3-(4-methoxybenzyloxy)-2-methylpentylcarbamate (11b) [ $\delta$ $1.14\left(3 \mathrm{H}, \mathrm{s}, \mathrm{CH}_{3}\right)$ ] and rac-benzyl (2R,3R)-2-hydroxy-3-(4-methoxybenzyloxy)-2methylpentylcarbamate (14b) $\left[\delta 1.11^{*}\left(3 \mathrm{H}, \mathrm{s}, \mathrm{CH}_{3}{ }^{*}\right)\right]$. Purification by flash chromatography (20\% ethyl acetate/ $n$-hexane) afforded an inseparable mixture of two diastereomers as a colorless oil (49.9 mg, 44\%), $R_{f} 0.33$ (40\% ethyl acetate/n-hexane). IR (thin film, $\mathrm{cm}^{-1}$ ) $3401(\mathrm{O}-\mathrm{H}, \mathrm{N}-\mathrm{H}), 3066$, 3033, 2966, 2932, 2877 (C-H), 1697 (C=O), 1529. ${ }^{1} \mathrm{H}-\mathrm{NMR}\left(400 \mathrm{MHz}, \mathrm{CDCl}_{3}\right.$ ) 7.27-7.37 (5H, m, ArH), 7.25 (2H, d, J 8.4 Hz, ArH), 6.86 (2H, d, J 8.8 Hz, ArH), 5.21 (1H, s, NH), 5.09-5.12 (2H, 
m, $\left.\mathrm{ArCH}_{2}\right), 4.65$ (1H, d, J $\left.10.4 \mathrm{~Hz}, \underline{\mathrm{C}}_{\mathrm{A}} \mathrm{H}_{\mathrm{B}}\right), 4.50$ (1H, d, J $\left.11.2 \mathrm{~Hz}, \mathrm{CH}_{\mathrm{A}} \underline{\mathrm{H}}_{\mathrm{B}}\right), 3.78$ (3H, s, OCH$)$ ), 3.35 (1H, dd, $J$ 14.0, 8.0 Hz, H1A), 3.26 (1H, dd, $J$ 8.8, 3.6 Hz, H3), 3.21 (1H, dd, $J$ 13.6, 3.6 Hz, H1B), 2.69 (1H, s, OH), 1.69 (1H, m, H4A), 1.55 (1H, m, H4B), 1.14 (3H, s, $\left.\mathrm{CH}_{3}\right)$ and 1.11* (3H, s, $\mathrm{CH}_{3}{ }^{*}$ ), 1.07 (3H, t, $J$ 7.6 Hz, H5) and 1.04* (3H, t, $J$ 7.6 Hz, H5 $\left.{ }^{*}\right) .{ }^{13} \mathrm{C}-\mathrm{NMR}\left(100 \mathrm{MHz}, \mathrm{CDCl}_{3}\right)$ 159.34, 157.31, 136.57, 130.39, 129.39, 128.47, 128.03, 127.97, 113.94, 86.90 (84.54*), 74.51, 74.42, 66.73 (66.94*), 55.24, 47.30 (48.08*), 23.69 (23.48*), $21.93\left(20.70^{*}\right), 11.82\left(11.29^{*}\right)$. LRMS $(\mathrm{ESI}+) 410\left([\mathrm{M}+\mathrm{Na}]^{+}, 100 \%\right), 121(16) . \mathrm{HRMS}(\mathrm{ESI}+)$ calcd for $\mathrm{C}_{22} \mathrm{H}_{29} \mathrm{NO}_{5} \mathrm{Na}\left([\mathrm{M}+\mathrm{Na}]^{+}\right)$ 410.1943, found 410.1938. ( ${ }^{*}$ Indicates signals of the minor diastereomer)

rac-(2S,3R)-1-(Benzyloxycarbonylamino)-2-hydroxy-2-methylpentan-3-yl acetate (12b) and rac-(2R,3R)-1-(benzyloxycarbonylamino)-2-hydroxy-2-methylpentan-3-yl acetate (15b)

The reaction was conducted according to general procedure 1 with rac-2-methylpent-1-en-3-yl acetate $(\mathbf{9 b})^{25}$ (56.0 $\left.\mathrm{mg}, 0.39 \mathrm{mmol}\right)$, potassium osmate dihydrate (5.80 $\mathrm{mg}, 0.02 \mathrm{mmol}$ ) and benzyl $N$-(4-tosyloxy)carbamate (1) (190 mg, $0.59 \mathrm{mmol}$ ). Purification by flash chromatography (30\% ethyl acetate/n-hexane) afforded rac-(2S,3R)-1-(benzyloxycarbonylamino)-2-hydroxy-2methylpentan-3-yl acetate (12b) as a colorless oil (67.0 mg, 55\%). $R_{f} 0.33$ (50\% ethyl acetate/nhexane). IR (thin film, $\left.\mathrm{cm}^{-1}\right) 3744(\mathrm{O}-\mathrm{H}), 3414(\mathrm{~N}-\mathrm{H})$, 3066, 3033, 2974, 2938, $2879(\mathrm{C}-\mathrm{H}), 1722$ (C=O), 1524. ${ }^{1} \mathrm{H}-\mathrm{NMR}$ (400 MHz, $\mathrm{CDCl}_{3}$ ) 7.29-7.36 (5H, m, ArH), 5.44 (1H, s, NH), 5.07-5.16 (2H, m, $\mathrm{CH}_{2} \mathrm{Ar}$ ), 4.80 (1H, dd, $J$ 10.4, $2.0 \mathrm{~Hz}, \mathrm{H} 3$ ), 3.38 (1H, dd, $J$ 14.0, 8.0 Hz, H1A), 3.01 (1H, dd, J 14.4, 4.8 Hz, H1B), 2.82 (1H, s, OH), 2.09 (3H, s, CH3), 1.80 (1H, m, H4A), 1.54 (1H, m, H4B), 1.15 (3H, s, CH3), 0.90 (3H, t, J 7.6 Hz, H5). ${ }^{13} \mathrm{C}-\mathrm{NMR}\left(100 \mathrm{MHz}, \mathrm{CDCl}_{3}\right.$ ) 171.68, 158.03, 136.28, 128.51, 128.14, 128.09, 78.16, 73.99, 67.08, 47.60, 21.66, 20.99, 20.92, 10.70. LRMS $(\mathrm{ESI}+) 332\left([\mathrm{M}+\mathrm{Na}]^{+}, 100 \%\right), 272$ (5), 91 (34). HRMS (ESI+) calcd for $\mathrm{C}_{16} \mathrm{H}_{23} \mathrm{NO}_{5} \mathrm{Na}\left([\mathrm{M}+\mathrm{Na}]^{+}\right)$ 332.1474, found 332.1487; calcd for $\mathrm{C}_{16} \mathrm{H}_{24} \mathrm{NO}_{5}\left([\mathrm{M}+\mathrm{H}]^{+}\right)$310.1654, found 310.1654.

A second fraction afforded rac-(2R,3R)-1-(benzyloxycarbonylamino)-2-hydroxy-2-methylpentan-3yl acetate (15b) as a colorless oil (23.8 mg, 20\%), $R_{f} 0.26$ (50\% ethyl acetate/ $n$-hexane). IR (thin film, $\left.\mathrm{cm}^{-1}\right) 3748(\mathrm{O}-\mathrm{H}), 3401(\mathrm{~N}-\mathrm{H})$, 3066, 3033, 2973, 2936, $2879(\mathrm{C}-\mathrm{H}), 1717(\mathrm{C}=\mathrm{O}), 1529 .{ }^{1} \mathrm{H}-$ NMR (400 MHz, $\left.\mathrm{CDCl}_{3}\right)$ 7.30-7.36 (5H, m, ArH), 5.23 (1H, s, NH), 5.11 (2H, s, $\left.\mathrm{CH}_{2} \mathrm{Ar}\right), 4.82$ (1H, dd, $J$ 10.4, 2.4 Hz, H3), 3.34 (1H, dd, J 14.0, 6.8 Hz, H1A), 3.11 (1H, dd, $J$ 14.0, 6.4 Hz, H1B), 2.43 (1H, s, OH), 2.13 (3H, s, CH3), 1.71 (1H, m, H4A), 1.58 (1H, m, H4B), 1.15 (3H, s, $\left.\mathrm{CH}_{3}\right), 0.89$ (3H, t, $J$ 7.2 Hz, H5). ${ }^{13} \mathrm{C}-\mathrm{NMR}\left(100 \mathrm{MHz}, \mathrm{CDCl}_{3}\right)$ 171.78, 157.06, 136.37, 128.51, 128.13, 128.06, 79.05, 74.45, 66.93, 48.08, 22.16, 20.92, 20.63, 10.48. LRMS (ESI+) 332 ([M+Na] $\left.]^{+}, 100 \%\right), 284$ (4), 272 (4), 91 (30). HRMS (ESI+) calcd for $\mathrm{C}_{16} \mathrm{H}_{23} \mathrm{NO}_{5} \mathrm{~K}\left([\mathrm{M}+\mathrm{K}]^{+}\right)$ 348.1213, found 348.1214; calcd for $\mathrm{C}_{16} \mathrm{H}_{23} \mathrm{NO}_{5} \mathrm{Na}\left([\mathrm{M}+\mathrm{Na}]^{+}\right)$332.1474, found 332.1474. 
rac-Benzyl (2S,3R)-2,3-dihydroxy-2-methylpentylcarbamate (13b) and rac-benzyl (2R,3R)-2,3dihydroxy-2-methylpentylcarbamate (16b)

Procedure 1: The reaction was conducted according to general procedure 1 with rac-2-methylpent1-en-3-ol (10b) $)^{24}$ (63.5 mg, $\left.0.63 \mathrm{mmol}\right)$, potassium osmate dihydrate (9.40 mg, $0.03 \mathrm{mmol}$ ) and benzyl $\mathrm{N}$-(4-tosyloxy)carbamate (1) (245 mg, $0.76 \mathrm{mmol}$ ). Integration of $\mathrm{C} 2-\mathrm{C}_{3} \underline{3}$ signal of the 400 $\mathrm{MHz}{ }^{1} \mathrm{H}-\mathrm{NMR}$ spectrum of the crude reaction mixture showed a 2.7:1 ratio of rac-benzyl (2S,3R)2,3-dihydroxy-2-methylpentylcarbamate (13b) [ $\delta 1.12$ (3H, s, $\left.\left.\mathrm{CH}_{3}\right)\right]$ and rac-benzyl (2R,3R)-2,3dihydroxy-2-methylpentylcarbamate (16b) [ $\delta 1.09$ (3H, s, $\left.\left.\mathrm{CH}_{3}\right)\right]$. Purification by flash chromatography (30\% ethyl acetate/n-hexane) afforded an inseparable mixture of diastereomers as a colorless oil (67.2 mg, 40\%), $R_{f} 0.36$ (70\% ethyl acetate/n-hexane). HRMS (ESI+) calcd for $\mathrm{C}_{14} \mathrm{H}_{21} \mathrm{NO}_{4} \mathrm{Na}\left([\mathrm{M}+\mathrm{Na}]^{+}\right)$290.1368, found 290.1368; calcd for $\left.\mathrm{C}_{14} \mathrm{H}_{21} \mathrm{NO}_{4} \mathrm{~K}(\mathrm{M}+\mathrm{K}]^{+}\right)$306.1108, found 306.1108.

Procedure 2: To rac-(2S,3R)-1-(benzyloxycarbonylamino)-2-hydroxy-2-methylpentan-3-yl acetate (12b) (25.0 mg, $0.08 \mathrm{mmol}$ ) was added a freshly prepared solution of potassium carbonate (22.4 $\mathrm{mg}, 0.16 \mathrm{mmol})$ in methanol $(3 \mathrm{ml})$ and water $(1 \mathrm{ml})$ at $20^{\circ} \mathrm{C}$. The reaction mixture was stirred until the reaction was complete $(1 \mathrm{~h})$. The reaction was quenched with aqueous hydrochloric acid $(5.0 \mathrm{ml}, 1.0 \mathrm{M})$, and extracted with dichloromethane $(3 \times 10 \mathrm{ml})$. The combined dichloromethane extract was washed with brine $(10 \mathrm{ml})$, dried over sodium sulfate and concentrated under reduced pressure to afford rac-benzyl (2S,3R)-2,3-dihydroxy-2-methylpentylcarbamate (13b) as a colorless oil (21.6 mg, 100\%), $R_{f} 0.37$ (70\% ethyl acetate/n-hexane). IR (thin film, $\mathrm{cm}^{-1}$ ) 3405 (broad, N-H, O-H), 3065, 3033, 2966, 2933, 2877 (C-H), 1696 (C=O), 1530. ${ }^{1} \mathrm{H}-\mathrm{NMR}$ (400 MHz, CDCl $)$ 7.307.37 (5H, m, ArH), 5.28 (1H, s, NH), 5.09-5.16 (2H, m, CH $2 \mathrm{Ar}), 3.50$ (1H, dd, J 14.8, $6.4 \mathrm{~Hz}, \mathrm{H3}$ ), 3.31 (1H, d, J 10.0 Hz, H1A), 3.09 (1H, dd, J 14.4, 6.0 Hz, H1B), 2.96 (1H, s, OH), 2.40 (1H, s, $\mathrm{OH}), 1.60$ (1H, m, H4A), 1.35 (1H, m, H4B), 1.12 (3H, s, CH3), 1.03 (3H, t, J 8.0 Hz, H5). ${ }^{13} \mathrm{C}-$ NMR (100 MHz, $\left.\mathrm{CDCl}_{3}\right)$ 158.08, 136.22, 128.54, 128.23, 128.10, 77.24, 74.62, 67.13, 47.50, 23.51, 20.75, 11.48. LRMS (ESI+) 290 ([M+Na $\left.]^{+}, 100 \%\right), 204$ (7), 91 (9). HRMS (ESI+) calcd for $\mathrm{C}_{14} \mathrm{H}_{21} \mathrm{NO}_{4} \mathrm{Na}\left([\mathrm{M}+\mathrm{Na}]^{+}\right)$290.1368, found 290.1360; calcd for $\mathrm{C}_{14} \mathrm{H}_{22} \mathrm{NO}_{4}\left([\mathrm{M}+\mathrm{H}]^{+}\right)$268.1549, found 268.1544 .

Procedure 3: To rac-(2R,3R)-1-(benzyloxycarbonylamino)-2-hydroxy-2-methylpentan-3-yl acetate (15b) (20.0 mg, $0.065 \mathrm{mmol}$ ) was added a freshly prepared of solution potassium carbonate (17.9 $\mathrm{mg}, 0.129 \mathrm{mmol})$ in methanol $(3 \mathrm{ml})$ and water $(1 \mathrm{ml})$ at $20^{\circ} \mathrm{C}$. The reaction mixture was stirred until the reaction was complete $(1 \mathrm{~h})$. The reaction was quenched with aqueous hydrochloric acid 
(5.0 ml, 1.0 M) and extracted with dichloromethane $(3 \times 10 \mathrm{ml})$. The combined dichloromethane extract was washed with brine $(10 \mathrm{ml})$, dried over sodium sulfate and concentrated under reduced pressure to afford rac-benzyl (2R,3R)-2,3-dihydroxy-2-methylpentylcarbamate (16b) as a colorless oil (16.7 mg, 97\%), $R_{f} 0.36$ (70\% ethyl acetate/n-hexane). IR (thin film, $\mathrm{cm}^{-1}$ ) 3400 (broad, N-H, O-H), 3066, 3033, 2964, 2931, 2876 (C-H), 1697 (C=O), 1530. ${ }^{1} \mathrm{H}-\mathrm{NMR}$ (400 MHz, CDCl $)$ 7.307.38 (5H, m, ArH), 5.22 (1H, s, NH), 5.11 (2H, s, CH $\mathrm{CH}_{2} \mathrm{Ar}$ ), 3.37 (1H, dd, J 14.0, 6.8 Hz, H3), 3.32 (1H, d, J 10.8 Hz, H1A), 3.12 (1H, dd, J 14.4, 6.0 Hz, H1B), 2.91 (1H, s, OH), 2.77 (1H, s, OH), 1.54 (1H, m, H4A), 1.40 (1H, m, H4B), 1.09 (3H, s, CH3), 1.00 (3H, t, J 7.2 Hz, H5). ${ }^{13} \mathrm{C}-\mathrm{NMR}$ (100 MHz, $\left.\mathrm{CDCl}_{3}\right)$ 157.65, 136.22, 128.54, 128.24, 128.10, 75.85, 74.45, 67.12, 48.60, 23.45, 20.24, 11.03. LRMS (ESI+) 290 ([M+Na] $\left.]^{+}, 100 \%\right), 204$ (4), 91 (5). HRMS (ESI+) calcd for $\mathrm{C}_{14} \mathrm{H}_{22} \mathrm{NO}_{4}\left([\mathrm{M}+\mathrm{H}]^{+}\right)$268.1549, found 268.1541; calcd for $\mathrm{C}_{14} \mathrm{H}_{21} \mathrm{NO}_{4} \mathrm{Na}\left([\mathrm{M}+\mathrm{Na}]^{+}\right)$290.1368, found 290.1364 .

\section{rac-Benzyl [(4S,5R)-5-ethyl-2,2,4-trimethyl-1,3-dioxolan-4-yl]methylcarbamate (18)}

The reaction was conducted according to general procedure 2 with rac-benzyl (2S,3R)-2,3dihydroxy-2-methylpentylcarbamate (13b) (21.8 mg, $0.08 \mathrm{mmol})$, 2,2-dimethoxypropane (42.5 mg, $0.41 \mathrm{mmol})$, camphorsulfonic acid $(0.800 \mathrm{mg}, 0.003 \mathrm{mmol})$ and $p$-toluenesulfonic acid (0.600 $\mathrm{mg}$, $0.003 \mathrm{mmol}$ ). The title compound 18 was isolated without further purification as a yellow oil (25.1 mg, 100\%), $R_{f} 0.51$ (40\% ethyl acetate/n-hexane). IR (thin film, $\mathrm{cm}^{-1}$ ) $3350(\mathrm{~N}-\mathrm{H}), 3066,3033$, 2981, 2935, 2878 (C-H), 1725 (C=O), 1513. ${ }^{1} \mathrm{H}-\mathrm{NMR}$ (400 MHz, $\mathrm{CDCl}_{3}$ ) 7.30-7.37 (5H, m, ArH), $5.11\left(2 \mathrm{H}, \mathrm{s}, \mathrm{CH}_{2} \mathrm{Ar}\right), 5.07$ (1H, s, NH), 3.73 (1H, dd, $J$ 8.8, $\left.4.0 \mathrm{~Hz}, \mathrm{H} 5\right), 3.31$ (1H, dd, $J$ 13.2, 8.8 Hz, H8A), 3.09 (1H, dd, J 13.6, 2.0 Hz, H8B), 1.46-1.65 (2H, m, H9), 1.40 (3H, s, CH3), 1.35 (3H, s, $\mathrm{CH}_{3}$ ), 1.23 (3H, s, $\mathrm{CH}_{3}$ ), 1.05 (3H, t, $\left.J 7.2 \mathrm{~Hz}, \mathrm{H} 10\right) .{ }^{13} \mathrm{C}-\mathrm{NMR}\left(100 \mathrm{MHz}, \mathrm{CDCl}_{3}\right)$ 156.81, 136.60, 128.46, 128.10, 128.03, 107.25, 84.62, 81.05, 66.73, 45.23, 28.32, 26.50, 21.70, 21.08, 11.44. LRMS (ESI+) 330 ([M+Na] $\left.]^{+}, 100 \%\right), 91$ (9). HRMS (ESI+) calcd for $\mathrm{C}_{17} \mathrm{H}_{26} \mathrm{NO}_{4}\left([\mathrm{M}+\mathrm{H}]^{+}\right)$ 308.1862, found 308.1865; calcd for $\mathrm{C}_{17} \mathrm{H}_{25} \mathrm{NO}_{4} \mathrm{Na}\left([\mathrm{M}+\mathrm{Na}]^{+}\right)$330.1681, found 330.1678.

\section{rac-Benzyl [(4R,5R)-5-ethyl-2,2,4-trimethyl-1,3-dioxolan-4-yl]methylcarbamate (19)}

The reaction was conducted according to general procedure 2 with rac-benzyl (2R,3R)-2,3dihydroxy-2-methylpentylcarbamate (16b) (16.7 mg, 0.06 mmol), 2,2-dimethoxypropane (32.5 mg, $0.31 \mathrm{mmol})$, camphorsulfonic acid $(0.600 \mathrm{mg}, 0.003 \mathrm{mmol})$ and $p$-toluenesulfonic acid (0.500 $\mathrm{mg}$, $0.003 \mathrm{mmol}$ ). The title compound 19 was isolated without further purification as a yellow oil (19.2 mg, 100\%), $R_{f} 0.46$ (40\% ethyl acetate/n-hexane). IR (thin film, $\mathrm{cm}^{-1}$ ) $3350(\mathrm{~N}-\mathrm{H}), 3066,3034$, 2981, 2933, 2879 (C-H), 1725 (C=O), 1516. ${ }^{1} \mathrm{H}-\mathrm{NNMR}$ (400 MHz, $\mathrm{CDCl}_{3}$ ) 7.30-7.37 (5H, m, 
ArH), 5.11 (3H, s, broad, $\mathrm{NH}$ and $\mathrm{CH}_{2} \mathrm{Ar}$ ), 3.66 (1H, dd, J 9.2, 4.0 Hz, H5), 3.31 (1H, dd, $J$ 14.0, 6.8 Hz, H8A), 3.20 (1H, dd, J 14.4, 5.6 Hz, H8B), 1.57 (1H, m, H9A), 1.47 (1H, m, H9B), 1.41 (3H, s, $\mathrm{CH}_{3}$ ), 1.31 (3H, s, $\left.\mathrm{CH}_{3}\right), 1.07$ (3H, s, $\mathrm{CH}_{3}$ ), 1.01 (3H, t, J 7.2 Hz, H10). ${ }^{13} \mathrm{C}-\mathrm{NMR}$ (100 $\mathrm{MHz}_{\mathrm{CDCl}}$ ) 156.60, 136.57, 128.47, 128.09, 128.01, 106.96, 81.83, 80.56, 66.77, 46.69, 28.60, 26.69, 22.42, 19.68, 11.18. LRMS (ESI+) 330 ([M+Na]+, 100\%), 91 (52). HRMS (ESI+) calcd for $\mathrm{C}_{17} \mathrm{H}_{26} \mathrm{NO}_{4}\left([\mathrm{M}+\mathrm{H}]^{+}\right)$308.1862, found 308.1861; calcd for $\mathrm{C}_{17} \mathrm{H}_{25} \mathrm{NO}_{4} \mathrm{Na}\left([\mathrm{M}+\mathrm{Na}]^{+}\right) 330.1681$, found 330.1681; calcd for $\mathrm{C}_{17} \mathrm{H}_{25} \mathrm{NO}_{4} \mathrm{~K}\left([\mathrm{M}+\mathrm{K}]^{+}\right)$346.1421, found 346.1420.

\section{rac-1-[(2,4-Dimethylpent-1-en-3-yloxy)methyl]-4-methoxybenzene (8c)}

To a stirred suspension of rac-2,4-dimethylpent-1-en-3-ol (10c) ${ }^{25}$ (480 mg, 4.20 mmol) and sodium hydride (202 mg, $8.41 \mathrm{mmol})$ in dimethylformamide $(3.0 \mathrm{ml}$ ) was added $p$-methoxybenzyl chloride (988 mg, $6.31 \mathrm{mmol}$ ) at room temperature under nitrogen. This reaction mixture was stirred until the reaction was complete. The reaction was quenched with aqueous sodium hydrogen carbonate $(5.0 \mathrm{ml}, 1.0 \mathrm{M})$ and extracted with dichloromethane $(3 \times 10 \mathrm{ml})$. The combined organic extract was washed with brine $(10 \mathrm{ml})$, dried over sodium sulfate and concentrated under reduced pressure to afford the crude product. Purification by flash chromatography (2\% ethyl acetate $/ n$-hexane) afforded the title compound 8c as a colorless oil (243 mg, 25\%), $R_{f} 0.23$ (5\% ethyl acetate/nhexane). IR (thin film, $\mathrm{cm}^{-1}$ ) 3070, 2957, 2870, 2836 (C-H), 1649, 1613, 1586 (C=C), 1514. ${ }^{1} \mathrm{H}-$ NMR (400 MHz, $\left.\mathrm{CDCl}_{3}\right) 7.23$ (2H, m, ArH), 6.85 (2H, m, ArH), 4.98 (1H, m, H1A), 4.85 (1H, m, H1B), 4.42 (1H, d, J 11.6 Hz, $\left.\underline{\mathrm{C}}_{\mathrm{A}} \mathrm{H}_{\mathrm{B}} \mathrm{Ar}\right), 4.13$ (1H, d, J $\left.11.6 \mathrm{~Hz}, \mathrm{CH}_{\mathrm{A}} \underline{\mathrm{H}_{\mathrm{B}}} \mathrm{Ar}\right), 3.79$ (3H, s, $\left.\mathrm{OCH}_{3}\right)$, 3.20 (1H, d, J 8.8 Hz, H3), 1.76 (1H, m, H4), 1.66 (3H, s, CH $\mathrm{CH}_{3}, 0.99$ (3H, d, J 6.4 Hz, CH $), 0.74$ (3H, d, $\left.J 6.8 \mathrm{~Hz}, \mathrm{CH}_{3}\right) .{ }^{13} \mathrm{C}-\mathrm{NMR}\left(100 \mathrm{MHz}, \mathrm{CDCl}_{3}\right)$ 143.78, 142.33, 131.06, 129.36, 114.82, 113.62, 89.30, 69.63, 55.23, 30.12, 19.51, 19.13, 16.61. LRMS (EI+) 234 (M+•, 100\%), 228 (80), 213 (12), 204 (22), 197 (54), 191 (70). HRMS (EI+) calcd for $\mathrm{C}_{15} \mathrm{H}_{22} \mathrm{O}_{2}\left(\mathrm{M}^{+\bullet}\right)$ 234.1620, found 234.1619.

rac-Benzyl (2S,3R)-2-hydroxy-3-(4-methoxybenzyloxy)-2,4-dimethylpentylcarbamate (11c) and rac-benzyl (2R,3R)-2-hydroxy-3-(4-methoxybenzyloxy)-2,4-dimethylpentylcarbamate (14c)

The reaction was conducted according to general procedure 1 with rac-1-[(2,4-dimethylpent-1-en3-yloxy)methyl]-4-methoxybenzene (8c) (84.5 mg, $0.36 \mathrm{mmol})$, potassium osmate dihydrate (5.30 mg, $0.02 \mathrm{mmol}$ ) and benzyl $N$-(4-tosyloxy)carbamate (1) (139 mg, $0.43 \mathrm{mmol}$ ). Purification by flash chromatography (30\% ethyl acetate/n-hexane) afforded an inseparable mixture of two diastereomers as a colorless oil (25.2 mg, 17\%), $R_{f} 0.28$ (40\% ethyl acetate/n-hexane). Purification 
by preparative HPLC ( $\mu$ Porasil silica $10 \mu \mathrm{m}, 3.9 \times 300 \mathrm{~mm}, 1 \%$ isopropanol $/ n$-hexane, flow rate 0.7 $\mathrm{ml} / \mathrm{min}$ ) gave a 12:1 ratio of diastereomers 11c and 14c and afforded rac-benzyl (2S,3R)-2-hydroxy3-(4-methoxybenzyloxy)-2,4-dimethylpentylcarbamate (11c) ( $\left.t_{\mathrm{R}} 23.7 \mathrm{~min}\right)$ as a colorless oil (21.8 mg, 15\%). IR (thin film, cm $\left.{ }^{-1}\right) 3421$ (O-H, N-H), 3065, 3032, 2959, 2874, 2837 (C-H), 1702 (C=O), 1612, 1586 (C=C), 1514. ${ }^{1} \mathrm{H}-\mathrm{NMR}$ (400 MHz, CDCl $)$ 7.29-7.38 (5H, m, ArH), 7.26 (2H, d, J $8.4 \mathrm{~Hz}, \mathrm{ArH}), 6.86$ (2H, d, J $8.4 \mathrm{~Hz}, \mathrm{ArH}), 5.16$ (1H, s, NH), 5.09 (2H, s, $\left.\mathrm{CH}_{2} \mathrm{Ar}\right), 4.68$ (1H, d, $\left.J 10.8 \mathrm{~Hz}, \underline{\mathrm{C}}_{\mathrm{A}} \mathrm{H}_{\mathrm{B}} \mathrm{Ar}\right), 4.46$ (1H, d, $\left.J 10.8 \mathrm{~Hz}, \mathrm{CH}_{\mathrm{A}} \underline{\mathrm{H}}_{\mathrm{B}} \mathrm{Ar}\right), 3.78$ (3H, s, $\left.\mathrm{OCH}_{3}\right), 3.37$ (1H, dd, $J$ 13.6, 8.0 Hz, H1A), 3.21 (1H, d, J 2.8 Hz, H3), 3.19 (1H, dd, J 11.6, 3.6 Hz, H1B), 2.68 (1H, s, OH), 1.99 (1H, m, H4), 1.17 (3H, s, CH3), 1.12 (3H, d, J 6.8 Hz, CH3), 1.03 (3H, d, J 6.8 Hz, H5). ${ }^{13} \mathrm{C}-$ NMR (100 MHz, $\mathrm{CDCl}_{3}$ ) 159.32, 157.28, 136.58, 130.36, 129.27, 128.47, 128.02, 127.96, 113.92, 89.27, 75.34, 74.90, 66.72, 55.23, 47.56, 28.96, 24.07, 22.18, 17.65. LRMS (ESI+) 424 ([M+Na] $]^{+}$, 100\%), 121 (90). HRMS (ESI+) calcd for $\mathrm{C}_{23} \mathrm{H}_{31} \mathrm{NO}_{5} \mathrm{Na}\left([\mathrm{M}+\mathrm{Na}]^{+}\right)$424.2100, found 424.2102; calcd for $\mathrm{C}_{23} \mathrm{H}_{32} \mathrm{NO}_{5}\left([\mathrm{M}+\mathrm{H}]^{+}\right)$402.2280, found 424.2290.

A second fraction afforded rac-benzyl (2R,3R)-2-hydroxy-3-(4-methoxybenzyloxy)-2,4dimethylpentylcarbamate (14c) $\left(t_{\mathrm{R}} 21.3 \mathrm{~min}\right)$ as a colorless oil (1.8 mg, 1.2\%). IR (thin film, $\mathrm{cm}^{-1}$ ) 3401 (O-H, N-H), 3031, 2958, 2925, 2872, 2852 (C-H), 1705 (C=O), 1612, 1587 (C=C), 1514. LRMS (ESI+) 424 ([M+Na]+, 100\%), 413 (11), 302 (32), 181 (8), 149 (18), 121 (65), 91 (42). HRMS (ESI+) calcd for $\mathrm{C}_{23} \mathrm{H}_{31} \mathrm{NO}_{5} \mathrm{Na}\left([\mathrm{M}+\mathrm{Na}]^{+}\right)$424.2100, found 424.2099.

rac-(2S,3R)-1-(Benzyloxycarbonylamino)-2-hydroxy-2,4-dimethylpentan-3-yl acetate (12c) and rac-(2R,3R)-1-(benzyloxycarbonylamino)-2-hydroxy-2,4-dimethylpentan-3-yl acetate (15c)

The reaction was conducted according to general procedure 1 with rac-2,4-dimethylpent-1-en-3-yl acetate (9c) $)^{25}$ (60.0 mg, $0.38 \mathrm{mmol}$ ), potassium osmate dihydrate (5.70 mg, $15.5 \mu \mathrm{mol}$ ) and benzyl $N$-(4-tosyloxy)carbamate (1) (148 mg, $0.46 \mathrm{mmol}$ ). Purification by flash chromatography (30\% ethyl acetate/n-hexane) afforded rac-(2S,3R)-1-(benzyloxycarbonylamino)-2-hydroxy-2,4dimethylpentan-3-yl acetate (12c) as a colorless oil (31.0 mg, 25\%), $R_{f} 0.34$ (40\% ethyl acetate/nhexane). IR (thin film, $\mathrm{cm}^{-1}$ ) 3416 (O-H, N-H), 3064, 3033, 2965, 2936, 2877 (C-H), 1722 (C=O), 1525. ${ }^{1} \mathrm{H}-\mathrm{NMR}\left(400 \mathrm{MHz}, \mathrm{CDCl}_{3}\right)$ 7.29-7.37 (5H, m, ArH), 5.36 (1H, s, NH), 5.08-5.16 (2H, m, $\mathrm{CH}_{2} \mathrm{Ar}$ ), 4.70 (1H, d, J 4.4 Hz, H3), 3.36 (1H, dd, J 14.4, 8.0 Hz, H1A), 3.04 (1H, dd, J 14.4, 4.4 Hz, H1B), 2.70 (1H, s, OH), 2.10-2.16 (4H, m, $\mathrm{CH}_{3}$ and H4), 1.19 (3H, s, $\left.\mathrm{CH}_{3}\right), 1.00$ (3H, d, J 6.8 $\left.\mathrm{Hz}, \mathrm{CH}_{3}\right), 0.94$ (3H, d, J 6.4 Hz, H5). ${ }^{13} \mathrm{C}-\mathrm{NMR}\left(100 \mathrm{MHz}, \mathrm{CDCl}_{3}\right)$ 171.35, 157.87, 136.34, 128.48, 128.11, 128.04, 79.95, 74.67, 67.03, 48.37, 28.14, 21.91, 21.53, 20.85, 18.27. LRMS (ESI+) 346 
([M+Na] $\left.]^{+}, 100 \%\right), 286(5), 91$ (11). HRMS (ESI+) calcd for $\mathrm{C}_{17} \mathrm{H}_{25} \mathrm{NO}_{5} \mathrm{Na}\left([\mathrm{M}+\mathrm{Na}]^{+}\right) 346.1630$, found 346.1623.

A second fraction afforded rac-(2R,3R)-1-(benzyloxycarbonylamino)-2-hydroxy-2,4dimethylpentan-3-yl acetate (15c) as a colorless oil (8.9 mg, 7\%), $R_{f} 0.20$ (40\% ethyl acetate/nhexane). IR (thin film, cm ${ }^{-1}$ ) 3400 (O-H, N-H), 3066, 3033, 2963, 2929, 2874 (C-H), 1721 (C=O), 1522. ${ }^{1} \mathrm{H}-\mathrm{NMR}\left(400 \mathrm{MHz}, \mathrm{CDCl}_{3}\right)$ 7.30-7.36 (5H, m, ArH), 5.24 (1H, s, NH), 5.11 (2H, s, $\mathrm{CH}_{2} \mathrm{Ar}$ ), 4.76 (1H, d, J 3.2 Hz, H3), 3.31 (1H, dd, J 14.0, 6.4 Hz, H1A), 3.02 (1H, dd, J 14.4, 6.4 Hz, H1B), 2.27 (1H, s, OH), 2.08-2.14 (4H, m, $\mathrm{CH}_{3}$ and $\mathrm{H} 4$ ), 1.21 (3H, s, $\mathrm{CH}_{3}$ ), 0.99 (3H, d, J 7.2 Hz, $\mathrm{CH}_{3}$ ), 0.94 (3H, d, J 6.4 Hz, H5). ${ }^{13} \mathrm{C}-\mathrm{NMR}$ (100 MHz, $\mathrm{CDCl}_{3}$ ) 171.45, 157.04, 136.40, 128.50, 128.12, 128.07, 79.95, 74.95, 66.89, 48.56, 28.32, 21.90, 21.31, 20.77, 17.04. LRMS (ESI+) $346\left([\mathrm{M}+\mathrm{Na}]^{+}\right.$, 100\%), 286 (7), 260 (5), 91 (8). HRMS (ESI+) calcd for $\mathrm{C}_{17} \mathrm{H}_{25} \mathrm{NO}_{5} \mathrm{Na}\left([\mathrm{M}+\mathrm{Na}]^{+}\right)$346.1630, found 346.1630.

rac-(3S,4R)-Benzyl 2,4-dihydroxy-2-methylpentan-3-ylcarbamate (25a) ${ }^{10}$

The reaction was conducted according to general procedure 1 with rac-4-methylpent-3-en-2-ol (20a) $)^{26}$ (45.0 mg, $0.45 \mathrm{mmol}$ ), potassium osmate dihydrate (6.60 mg, $0.02 \mathrm{mmol}$ ) and benzyl $\mathrm{N}$-(4tosyloxy)carbamate (1) (173 mg, $0.54 \mathrm{mmol})$. Purification by flash chromatography (80\% diethyl ether/n-hexane) afforded the title compound 25a as a brown oil (11.3 $\mathrm{mg}, 9.4 \%), R_{f} 0.14$ (80\% diethyl ether/n-hexane). IR (thin film, $\mathrm{cm}^{-1}$ ) 3414 (broad, O-H, N-H), 3067, 3033, 2956, 2923, 2852 (C-H), 1721 (C=O), 1699, 1513. ${ }^{1} \mathrm{H}-\mathrm{NMR}$ (400 MHz, CDCl $)$ 7.31-7.37 (5H, m, ArH), 5.56 (1H, d, J 9.6 Hz, NH), 5.14 (2H, s, CH $2 \mathrm{Ar}), 4.42$ (1H, q, J 5.6 Hz, H4), 3.37 (1H, d, J 9.2 Hz, H3), 2.59 (2H, s, broad, $2 \times \mathrm{OH}), 1.38$ (3H, s, $\left.\mathrm{CH}_{3}\right), 1.24$ (3H, s, H1), 1.18 (3H, d, J 6.4 Hz, H5). ${ }^{13} \mathrm{C}-$ NMR (100 MHz, $\left.\mathrm{CDCl}_{3}\right)$ 157.35, 136.57, 128.50, 128.06, 127.86, 74.49, 67.16, 66.84, 60.54, 27.87, 27.65, 20.56. LRMS (ESI+) 290 ([M+Na]+, 85\%), 272 (28), 245 (11), 232 (5), 177 (5), 149 (6), 132 (10), 91 (100), 89 (9), 57 (5). HRMS (ESI+) calcd for $\mathrm{C}_{14} \mathrm{H}_{21} \mathrm{NO}_{4} \mathrm{Na}\left([\mathrm{M}+\mathrm{Na}]^{+}\right)$290.1368, found 290.1367.

\section{rac-(3S,4R)-Benzyl 2,4-dihydroxy-2-methylhexan-3-ylcarbamate (25b)}

The reaction was conducted according to general procedure 1 with rac-5-methylhex-4-en-3-ol (20b) $)^{26}$ (50.0 mg, $0.44 \mathrm{mmol}$ ), potassium osmate dihydrate (6.50 mg, $0.02 \mathrm{mmol}$ ) and benzyl $\mathrm{N}$-(4tosyloxy)carbamate 1 (169 mg, $0.53 \mathrm{mmol}$ ). Purification by flash chromatography (80\% diethyl ether/ $n$-hexane) afforded the title compound $\mathbf{2 5 b}$ as a brown oil ( $8.2 \mathrm{mg}, 6.7 \%), R_{f} 0.33$ (80\% diethyl ether/n-hexane). IR (thin film, cm ${ }^{-1}$ ) 3406 (broad, O-H, N-H), 2970, 2934 (C-H), 1694 (C=O), 1515. ${ }^{1} \mathrm{H}-\mathrm{NMR}\left(400 \mathrm{MHz}, \mathrm{CDCl}_{3}\right.$ ) 7.29-7.36 (5H, m, ArH), 5.52 (1H, d, J $10.0 \mathrm{~Hz}, \mathrm{NH}$ ), 
5.09-5.16 (2H, m, CH $2 \mathrm{Ar}), 4.08$ (1H, t, J 7.2 Hz, H4), 3.47 (1H, dd, J 9.6, 1.6 Hz, H3), 2.67 (1H, s, OH), 2.54 (1H, s, OH), 1.54-1.47 (2H, m, H5), 1.38 (3H, s, CH3), 1.25 (3H, s, H1), 0.95 (3H, t, J 7.6 Hz, H6). ${ }^{13} \mathrm{C}-\mathrm{NMR}\left(100 \mathrm{MHz}, \mathrm{CDCl}_{3}\right)$ 157.22, 136.57, 128.48, 128.05, 127.87, 74.60, 72.92, 66.82, 58.84, 27.84, 27.67, 27.32, 9.98. LRMS (ESI+) 304 ([M+Na]+, 100\%), 286 (4), 145 (5), 91 (84), 85 (5). HRMS (ESI+) calcd for $\mathrm{C}_{15} \mathrm{H}_{23} \mathrm{NO}_{4} \mathrm{Na}\left([\mathrm{M}+\mathrm{Na}]^{+}\right)$304.1525, found 304.1526.

\section{Results}

The choice of substrates was influenced by practical considerations. In the absence of ligand control, the intermolecular oxyamination of unsymmetrically substituted alkenes by pre-formed nitrogen sources typically gives high selectivity for products where the nitrogen adds to the less substituted end of the double bond. ${ }^{4,9-12}$ High regioselectivity in the oxyamination reaction would significantly simplify product analysis of the diastereomeric mixtures. On this basis, the oxyamination reaction of mono-, 1,1-di- and tri-substituted alkenes expected to display high regioselectivity was investigated.

A similar procedure was employed for the oxyamination of all allylic alcohol derivatives. ${ }^{12}$ This involved reaction of the allylic alcohol or its derivative (1.0 equiv) and benzyl $\mathrm{N}$-(4tosyloxy)carbamate 1 (1.2 equiv) with potassium osmate dihydrate (0.04 equiv) in acetonitrile/water (3:1). The reaction diastereoselectivity was determined by $400 \mathrm{MHz}{ }^{1} \mathrm{H}$ NMR integration of the crude reaction mixture, or where this was not possible by HPLC-UV integration or by product isolation after careful purification by flash chromatography.

The yields and diastereoselectivities for the oxyamination of a range allylic alcohol derivatives 2, $\mathbf{3}$, 8-10 containing mono- and 1,1-di-substituted double bonds are presented in Tables I and II respectively. Several trends were observed. The amino alcohol products 4-7 and 11-16 were afforded in moderate to good combined yield but long reaction times were normally required. Lower yields were afforded from more highly substituted substrates and also those bearing unprotected allylic hydroxyl groups. While the oxyamination of allylic alcohol 10c failed to afford product under the standard conditions, reaction of the corresponding para-methoxybenzyl and acetate protected derivatives $\mathbf{8 c}$ and $\mathbf{9 c}$ did afford amino-alcohol products, albeit in low yields (Table II, Entries 3, 6 and 9). High regioselectivity was achieved as expected, with the terminal amine product produced exclusively from both protected and unprotected allylic alcohol derivatives. 
In all cases examined, the anti diastereomers 4, $\mathbf{5}$ and 11-13 were the major products of the oxyamination reaction, with anti:syn selectivities ranging from 1.2:1 to 12:1. Increasing the size of the alkyl substituent appended to the allylic alcohol-bearing stereogenic center was observed to modestly increase selectivity for the anti diastereomer. For example, allylic alcohol 3a with methylsubstituent provided a 1.8:1 / anti:syn ratio while the reaction of allylic alcohol 3c with isopropyl substituent gave a 3.1:1 / anti:syn ratio (Table I, Entries 4-6). The 1,1-disubstituted alkenes 8-10 (Table II, Entries 4-8) afforded higher selectivity than their corresponding monosubstituted counterparts 2, 3 (Table I, Entries 1-5). No significant change in diastereoselectivity was observed on protection of the alcohols as the corresponding acetate esters (Table I, Entries 1-6 and Table II, Entries 4-8). However, improved anti selectivity was observed for the para-methoxybenzyl ethers examined (Table II, Entries 1-3).

\section{$<$ Table I here $>$ \\ $<$ Table II here >}

The relative configuration of anti acetate $\mathbf{4 a}$ and anti diol 5a was confirmed by a sequence of deacetylation followed by acetonide formation (Scheme 2). The $400 \mathrm{MHz}{ }^{1} \mathrm{H}$ NMR spectrum revealed two acetonide methyl signals at $\delta 1.44$ and $\delta 1.33$ consistent with a cis-4,5-disubstituted2,2-dimethyldioxolane $17 .{ }^{27}$ In addition, irradiation of $\mathrm{H} 5(\delta 4.33$, dq, $J 6.3,6.3 \mathrm{~Hz}$ ) showed nOes to $\mathrm{H} 4(\delta 4.11, \mathrm{~m})$ and the $\alpha$-oriented acetonide methyl signal $(\delta 1.33, \mathrm{~s}){ }^{27}$

\section{$<$ Scheme 2 here $>$}

Scheme 2. Stereochemical proof for acetate $\mathbf{4 a}$ and diol $\mathbf{5 a .}$

The relative configuration of acetates $\mathbf{1 2 b}$ and $\mathbf{1 5 b}$ and their corresponding diols $\mathbf{1 3 b}$ and $\mathbf{1 6 b}$ were confirmed by a sequence of deacetylation followed by acetonide formation (Scheme 3). The anti acetate $\mathbf{1 2 b}$ afforded anti diol $\mathbf{1 3 b}$ and acetonide 18. For the acetonide 18, irradiation of H5 ( $\delta 3.73$, dd, $J$ 8.8, $4.0 \mathrm{~Hz}$ ) showed nOes to the C4-methyl ( $\delta 1.23$, s) and the $\alpha$-oriented acetonide methyl signal $(\delta 1.35, \mathrm{~s})$. The diastereomeric syn acetate $\mathbf{1 5 b}$ afforded syn diol $\mathbf{1 6} \mathbf{b}$ and acetonide $\mathbf{1 9}$. For the acetonide 19, irradiation of $\mathrm{H} 5(\delta 3.66$, dd, $J 9.2,4.0 \mathrm{~Hz})$ showed nOes to H8A $(\delta 3.31$, dd, $J$ $14.0,6.8 \mathrm{~Hz})$ and the $\alpha$-oriented acetonide methyl signal $(\delta 1.31, \mathrm{~s})$. The relative configuration of anti benzyl ether 11a was confirmed through selective deprotection of the para-methoxybenzyl ether and comparison with the previously described anti diol 13a (Scheme 4). ${ }^{12}$

$<$ Scheme 3 here $>$ 
Scheme 3. Stereochemical proof for acetates 12b, 15b and diols 13b, $\mathbf{1 6 b}$.

$<$ Scheme 4 here $>$

Scheme 4. Stereochemical proof for PMB ether 11a.

The reactivity of a range of trisubstituted alkenes $\mathbf{2 0 - 2 3}$ is presented in Table III. In contrast to the allylic alcohol derivatives bearing mono- and 1,1-disubstituted double bonds, the oxyamination reaction compounds bearing trisubstituted double bonds failed to afford the products 24-31 in the majority of cases investigated. In two cases examined oxyamination products were observed in low yields to give the syn diastereomer 25a,b (Table III, Entries 1 and 2) as the major product isolated. The identity and relative configuration of compound 25a was confirmed by comparison with the literature. ${ }^{10}$ The reactions of these alkenes were characterised by the formation of a number of unidentified by-products. Longer reaction times did not afford higher yields of product.

$<$ Table III here>

\section{Discussion}

The pioneering work conducted by Kishi and co-workers provided an empirical rule for the observed diastereoselectivity of the osmium tetroxide mediated dihydroxylation of allylic alcohol derivatives. ${ }^{18,19,28}$ Since this time, several important experimental studies delineating the scope and limitations of this phenomenon have been reported, ${ }^{29-31}$ and a series of refined models have been advanced to explain the observed diastereoselectivity. ${ }^{20,29-31}$ The model advanced by Houk and coworkers and developed with the aid of DFT computation predicts the anti diastereomer as the major product of the dihydroxylation of $(E)$-3-buten-2-ol with ligated osmium tetroxide. ${ }^{20}$ Adapting this model to the oxyamination reaction of allylic alcohol derivatives bearing mono- and 1,1disubstituted double bonds is shown in Scheme 5. The imidoosmium catalyst approaches the alkene with the large imido-group directed toward the unsubstituted and sterically unencumbered terminus of the double bond leading to high regioselectivity. The favored approach of the imidoosmium catalyst to the si-face of the double bond positions the allylic oxygen substituent (OP) in the inside position to avoid unfavorable electrostatic and stereoelectronic effects ${ }^{20}$ and the large alkyl substituent (R) in the sterically unencumbered anti position giving transition structure $\mathbf{A}$ and leading to the anti diastereomer. In contrast, approach of the imidoosmium catalyst to the re-face of the double bond with the allylic oxygen substituent (OP) in the inside position leaves the large alkyl substituent (R) in the more sterically encumbered outside position giving transition structure $\mathbf{B}$ and leading to the syn diastereomer. Consistent with this proposal, the results show a modest but 
consistent and systematic trend of increasing selectivity for the anti diastereomer as the alkyl substituent (R) on the allylic stereocenter increases in size from methyl to isopropyl, reinforcing the preference for the less sterically encumbered transition structure A (Tables I and II). Furthermore, the introduction of a methyl substituent ( $\left.\mathrm{R}^{\prime}\right)$ on the double bond also leads to an increase in anti selectivity due to increased steric interactions between this substituent ( $\left.\mathrm{R}^{\prime}\right)$ and the alkyl substituent (R) on the allylic stereocenter in transition structure B (compare Table I, Entries 1-5 and Table II, Entries 4-8 respectively).

$<$ Scheme 5 here $>$

Scheme 5. Proposed transition structures for the oxyamination of allylic alcohol derivatives bearing mono- and 1,1-disubstituted double bonds.

Similar trends in stereoselectivity have been observed for the osmium catalyzed dihydroxylation of allylic alcohol derivatives. ${ }^{18,19,28,30,31}$ In particular, the sense and magnitude of diastereoselectivity noted by Evans and Kaldor ${ }^{31}$ for the osmium-catalyzed dihydroxylation of allylic benzyl ether substrates 26b,c bearing 1,1-disubstituted double bonds (Scheme 6) parallels the results of this work for the oxyamination of structurally related para-methoxybenzyl ether derivatives (Table II, Entries 1-3). The isopropyl derivative $\mathbf{8 c}$ gave the highest anti selectivity of the terminal alkene substrates examined.

$<$ Scheme 6 here $>$

Scheme 6. Evans dihydroxylation ${ }^{31}$ and oxyamination of allylic benzyl ether derivatives bearing 1,1-disubstituted double bonds.

The osmium-catalyzed oxyamination of alkene 20a (Table III, Entry 1) unexpectedly afforded the syn diastereoisomer 25a as the major product identified in low yield in contrast to the anti diastereoisomers observed for the terminal alkene substrates (Tables I and II). A related oxyamination protocol was recently reported to afford the same product. ${ }^{10}$ The anti diastereomer 24a was not observed. Kishi's empirical rule for the dihydroxylation of allylic alcohol derivatives with cis-substituted double bonds predicts anti selectivity, as do other models of diastereoselectivity, ${ }^{18,19,29,30}$ including the aforementioned model advanced by Houk and co-workers which predicts the anti diastereomer as the major product of the dihydroxylation of (Z)-3-buten-2-ol with ligated osmium tetroxide. ${ }^{20}$ Several features of this oxyamination reaction are noteworthy. The double bond substitution pattern of the trisubstituted alkenes 20a,b favor addition of the large $N$ (benzyloxycarbonyl)imido-osmium grouping to the least substituted carbon of the double bond, in 
close proximity to the allylic stereocenter. This contrasts with the oxyamination of terminal alkenes where a smaller oxo-osmium group occupies this proximal position (Scheme 5). Furthermore, the oxyamination reactions of trisubstituted alkenes occurred in low yields ${ }^{10,12}$ and in most cases examined in this study failed to afford product under the standard conditions. Given the low reactivity observed and the limited data available, further experimentation is warranted before drawing conclusions on the basis for, and generality of, this stereochemical outcome.

\section{Conclusion}

In conclusion, the diastereoselective oxyamination reaction of chiral allylic alcohol derivatives bearing mono- or 1,1-di-substituted double bonds was observed to occur in moderate to good yield, excellent regioselectivity, and with moderate substrate controlled anti diastereoselectivity. The observed stereoselectivity shows a clear and systematic trend with anti:syn ratios increasing in line with substituent size at both the allylic stereocenter and double bond $\alpha$-carbon. The stereoinduction is in accord with the sense of diastereoselectivity predicted by Kishi's empirical rule, and a previously reported transition state model for the osmium-catalyzed dihydroxylation of allylic alcohol derivatives. In contrast, the osmium mediated oxyamination of allylic alcohol derivatives bearing trisubstituted double bonds displayed low or no reactivity and provided the syn diastereoisomer in two examples. These results highlight the potential for the use of substratecontrolled oxyamination in the synthesis of stereochemically complex natural products and other amino alcohol containing compounds.

\section{Acknowledgements}

We thank the Directorate General of Higher Education Indonesia (DIKTI) for scholarship support and Tony Herlt (ANU) for assistance with HPLC.

\section{References}

1. Donohoe TJ, Callens CKA, Flores A, Lacy AR, Rathi AH. Recent developments in methodology for the direct oxyamination of olefins. Chem Eur J 2011;17:58-76.

2. Bergmeier SC. The synthesis of vicinal amino alcohols. Tetrahedron 2000;56:2561-2576.

3. Li G, Chang H-T, Sharpless KB. Catalytic asymmetric aminohydroxylation (AA) of olefins. Angew Chem Int Ed Engl 1996;35:451-454. 
4. Bodkin JA, McLeod MD. The Sharpless asymmetric aminohydroxylation. J Chem Soc Perkin Trans 1 2002;2733-2746.

5. Donohoe TJ, Callens CKA, Lacy AR, Winter C. Tethered aminohydroxylation reaction and its application to total synthesis. Eur J Org Chem 2012:655-663.

6. Donohoe TJ, Chughtai MJ, Klauber DJ, Griffin D, Campbell AD. $N$-sulfonyloxy carbamates as reoxidants for the tethered aminohydroxylation reaction. J Am Chem Soc 2006;128:25142515.

7. Donohoe TJ, Bataille CJR, Gattrell W, Kloesges J, Rossignol E. Tethered aminohydroxylation: dramatic improvements to the process. Org Lett 2007;9:1725-1728.

8. Donohoe TJ, Callens CKA, Thompson AL. Tethered aminohydroxylation (TA) reaction of amides. Org Lett 2009;11:2305-2307.

9. Harris L, Mee SPH, Furneaux RH, Gainsford GJ, Luxenburger A. Alkyl 4chlorobenzoyloxycarbamates as highly effective nitrogen source reagents for the base-free, intermolecular aminohydroxylation reaction. J Org Chem 2011;76:358-372.

10. Ma Z, Naylor BC, Loertscher BM, Hafen DD, Li JM, Castle SL. Regioselective base-free intermolecular aminohydroxylations of hindered and functionalized alkenes. J Org Chem 2012;77:1208-1214.

11. Donohoe TJ, Callens CKA, Flores A, Mesch S, Poole DL, Roslan IA. Amino acid-based reoxidants for aminohydroxylation: application to the construction of amino acid-amino alcohol conjugates. Angew Chem Int Ed 2011;50:10957-10960.

12. Masruri, Willis AC, McLeod MD. Osmium-catalyzed vicinal oxyamination of alkenes by $N$ (4-toluenesulfonyloxy)carbamates. J Org Chem 2012;77:8480-8491.

13. Pringle W, Sharpless KB. The osmium-catalyzed aminohydroxylation of Baylis-Hillman olefins. Tetrahedron Lett 1999;40:5151-5154.

14. Donohoe TJ, Lacy AR, Rathi AH, Walter DS. The influence of exocyclic stereochemistry on the tethered aminohydroxylation reaction. Chem Asian J 2011;6:3214-3222.

15. Bedoya Zurita M, Ahond A, Poupat C, Potier P. Première synthèse totale de la girolline. Tetrahedron 1989;45:6713-6720. 
16. Hirano S, Ichikawa S, Matsuda A. Total synthesis of caprazol, a core structure of the caprazamycin antituberculosis antibiotics. Angew Chem Int Ed 2005;44:1854-1856.

17. Hirano S, Ichikawa S, Matsuda A. Development of a highly $\beta$-selective ribosylation reaction without using neighboring group participation: total synthesis of $(+)$-caprazol, a core structure of caprazamycins. J Org Chem 2007;72:9936-9946.

18. Cha JK, Christ WJ, Kishi Y. On stereochemistry of osmium tetroxide oxidation of allylic alcohol systems: empirical rule. Tetrahedron Lett 1983;24:3943-3946.

19. Cha JK, Christ WJ, Kishi Y. On stereochemistry of osmium tetraoxide oxidation of allylic alcohol systems. Empirical rule. Tetrahedron 1984;40:2247-2255.

20. Haller J, Strassner T, Houk KN. Models for stereoselective additions to chiral allylic ethers: osmium tetroxide bis-hydroxylations. J Am Chem Soc 1997;119:8031-8034.

21. Vogt M, Ceylan S, Kirschning A. Stereocontrolled palladium-catalysed umpolung allylation of aldehydes with allyl acetates. Tetrahedron 2010;66:6450-6456.

22. Hoekman MJ, Fagan GL, Webb AD, Kepner RE. Synthesis of homologs of 4,5-dihydroxyand 4-hydroxy-5-oxohexanoic acid $\gamma$-lactones. J Agric Food Chem 1982;30:920-924.

23. Wennerberg J, Olofsson C, Frejd T. Zeolite $\beta$-induced rearrangement of alkoxybenzyl allyl ethers to aldehydes and ketones. 5. Variation of the allylic moiety. J Org Chem 1998;63:35953598.

24. Cossy J, Bauer D, Bellosta V. Formal total synthesis of (+)-methynolide. Tetrahedron 2002;58:5909-5922.

25. Barile F, Bassetti M, D’Annibale A, Gerometta R, Palazzi M. Cross-metathesis reactions of homoallyl methyl malonates with sterically hindered allylic esters. Eur J Org Chem 2011:6519-6526.

26. Adam W, Peters K, Peters EM, Stegmann VR. Hydroxy-directed regio- and diastereoselective [2+2] photocycloaddition (Paternò-Büchi reaction) of benzophenone to chiral allylic alcohols. J Am Chem Soc 2000;122:2958-2959.

27. Sable HZ, Ritchey WM, Nordlander JE. Studies on cyclitols. XIV. Methyl group signals of gem-dimethyldioxolanes as indicators of anisotropy. Spectrosc Lett 1969;2:239-245. 
28. Cha JK, Kim N-S. Acyclic stereocontrol induced by allylic alkoxy groups. Synthetic applications of stereoselective dihydroxylation in natural product synthesis. Chem Rev 1995;95:1761-1795.

29. Houk KN, Duh HY, Wu YD, Moses SR. Steric models for stereoselectivity of nitrile oxide cycloadditions to chiral alkenes. J Am Chem Soc 1986;108:2754-2755.

30. Vedejs E, McClure CK. Hyperconjugative effects of allylic substituents are not important in osmylations. J Am Chem Soc 1986;108:1094-1096.

31. Evans DA, Kaldor SW. The stereoselective osmylation of 1,1-disubstituted olefins. Effect of allylic substituents on reaction diastereoselectivity. J Org Chem 1990;55:1698-1700. 